\title{
A Dynamic Specification to Automatically Debug and Correct Various Divider Circuits
}

\author{
Mohammad Hashem Haghbayan, Student Member, and Bijan Alizadeh*, Senior Member, IEEE \\ * Corresponding author \\ School of Electrical and Computer Engineering, College of Engineering, University of Tehran, Tehran, Iran \\ h.haghbayan@ece.ut.ac.ir; b.alizadeh@ut.ac.ir
}

\begin{abstract}
This paper presents a formal technique to verify and debug division circuits on fixed point numbers. The proposed technique is based on a reverse-engineering mechanism of obtaining a high level model of the gate level implementation and also introducing an intermediate representation of the specification that makes equivalence checking between two models possible. The main advantage of this representation is the fact that the specification is dynamically updated according to the information obtained from the implementation. At the end, if two updated models are not equivalent, possible bugs can be localized and then corrected automatically by analyzing the difference, if possible. Experimental results show the robustness of the proposed technique in comparison with other contemporary methods in terms of the run time and also show that two orders of magnitude average speedup is obtained.
\end{abstract}

Index Terms-Arithmetic and logic units, Debugging aids, Diagnostics, Verification, Formal models

\section{INTRODUCTION}

$\mathrm{A}$ $\mathrm{S}$ the size and complexity of digital systems increase continuously, design verification and debug are quickly becoming more important. From a verification point of view, one of the most difficult parts in such complicated designs is arithmetic datapaths and their components, such as multipliers and dividers. Most of hardware verification tools are based on bitlevel methods like Binary Decision Diagrams (BDDs) or Satisfiability (SAT) solvers that are not scalable because they suffer from space and time explosion problems when dealing with large arithmetic circuits [1][2]. To alleviate this problem, word-level Decision Diagrams like Binary Moment Diagrams (BMDs), *BMDs and $\mathrm{K} * \mathrm{BMDs}$ have been proposed [3]. However, these diagrams and decision procedures still suffer from the memory explosion when dealing with wide ranges of arithmetic operations due to the fact that functions are defined over binary variables as a bit vector rather than integer variables. Another methodology transforms arithmetic circuits into propositional logic formulas and then satisfiability tools, i.e., SAT or Sat-Modulo Theories (SMT) solvers, are employed to verify the validity of the formulas [11]. Although Boolean SATbased methods are very efficient regarding memory requirement, they have not been very successful for designs containing large arithmetic units due to computational complexity. SMT-based verification techniques are also suffering from the same problem because when dealing with arithmetic circuits most SMT solvers resort to bit blasting and therefore they show the same performance limitations as pure SAT solvers [11]. In [24] the authors proposed a SAT-based solution for logic diagnosis of multiple faults or design errors in combinational and sequential circuits. Again the proposed method is not scalable in terms of time and memory space since SAT solver is used for the verification and diagnosis process. In addition, such decision diagrams and decision procedures are not able to handle division circuits which are one of the most important operations of arithmetic computations.

A well-known approach in verifying arithmetic circuits is to extract arithmetic operations from the gate-level implementation and then generate an arithmetic model to be compared with a high level specification [5][10]. For example this technique can check the equivalence of integer multipliers based on a bit level reverse-engineering approach. The main challenge is to efficiently extract an arithmetic bit level description of a circuit from a given gate-level arithmetic circuit. Reverse engineering could be considered as a very pragmatic approach to multiplier verification. As the number of possible architectures for a multiplier is limited one may incorporate a variety of architectures in the frontend of the equivalence checker and repeat the comparison for all of them. The verification approach in [5][10] benefits from an efficient reverse-engineering process in extracting a network of half-adders from the gate-level circuit, while there is no need of an exhaustive process to map carry signals.

The authors of [4] proposed a formal method to describe and verify arithmetic circuits using symbolic computer algebra. The main idea is to describe arithmetic circuits with integer equations in a hierarchical manner. These equations (potentially very large) are formally verified by formula manipulations based on Groebner basis [7]. The authors of [12] modeled a gate level implementation by polynomials over rings $Z_{2}{ }^{n}$ and then the normal forms are computed with respect to the Groebner basis using modern computer algebra algorithms. This model is complicated and is not scalable to practical designs. The authors of [13], take the advantage of Groebner basis theory to verify arithmetic circuits over rings $\mathrm{Z}_{2}{ }^{\mathrm{n}}$. Instead of mapping each gate to a polynomial, they have significantly reduced the number of polynomials by finding fanout-free regions and representing the whole region by one single polynomial. Although these techniques seem to be robust for large arithmetic circuits, they are not able to handle dividers. One of our contributions in this paper is extending the debugging technique in [5][10] in order to verify and debug large arithmetic systems that contain dividers in addition to adders and multipliers.

There has been considerable interest in using theorem proving techniques to verify arithmetic circuits such as multipliers and dividers [8][14][15]. In these techniques, gate level implementation and high level specification should be translated into or described in an appropriate specification language which is usually equation-based. Then, a proof by induction is attempted to check the equivalence between two models that needs user guidance. Symbolic Trajectory Evaluation (STE) based formal verification approach has been widely used at Intel in the past for various microprocessor designs to formally 
verify data-path designs [20]. The authors of [21] implement industrial level tools tied together in the ACL2 theorem prover and focus on hardware validation. Although such techniques are scalable enough to be able to verify large arithmetic circuits, their main drawback is to balance efficiency with generality since a single proof strategy is being applied to all theorems. In other words, the main problem with theorem proving techniques is the lack of expertise and documentation. It takes a considerably long time to learn theorem proving techniques and also there is a strong need for libraries of specifications to be established, and more automated tools and approaches.

In this work, we assume that a gate-level implementation as well as a high level specification of a complex arithmetic datapath on fixed point numbers is given. Fig. 1 shows the basic idea behind the proposed debugging technique. As can be seen in the figure, from the specification, expected arithmetic operations are extracted and represented using functional bit level adder (FBLA) representation. FBLA is an extension of the BLA in [5][10] that will be explained in Section III. Based on such a model, adders and other controlling logics are extracted from the gate-level implementation and then represented using logical bit level adder (LBLA) as will be discussed in Section IV. During the extraction of high level components from the implementation, FBLA will be updated if needed. This dynamic updating mechanism allows the algorithm to prepare FBLA for various structures based on extracted adders or repetitive logic cones. Then, the equivalence between the logics extracted from the implementation and expected arithmetic operations extracted from the specification are checked using a canonical mixed bit- and word-level decision diagram called Horner Expansion Diagram (HED) [16]. In the case of non-equivalent functionalities, error correction is performed by replacing buggy logics with expected functions from the functional bit level model. It should be noted that the expected functions in FBLA model are gradually updated during processing the implementation and cannot be specified independent of the implementation.

In addition to the above-mentioned methods, there are many methods for verification of dividers specially SRT dividers [1][17][18][19]. In general most of them cannot support optimization techniques and in some cases the run time is very high.

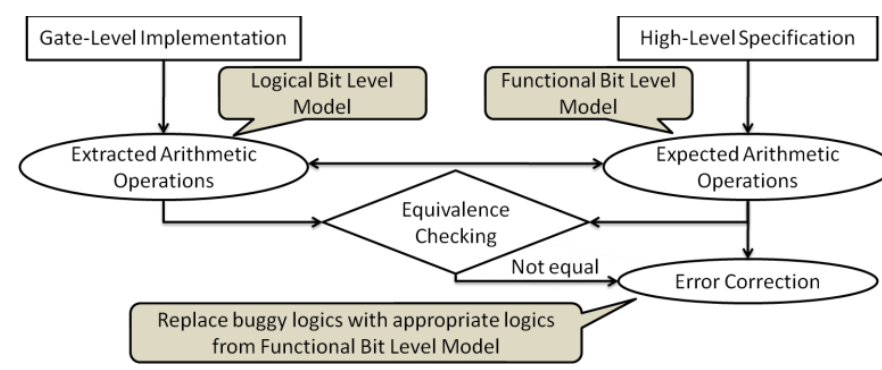

Figure 1. Basic idea of the proposed debugging and correction techniques

In summary, the main contributions of this paper in comparison with our previous works [5][10][23] are as follows:

- An algorithm tightly integrating verification and debugging of fast array dividers and sequential dividers even in the case of speeding up the remainder update stage (quotient selector logic) in the implementation where the design makes use of a sum and carry representation for the remainder, as will be discussed in Section IV.
- Dynamically representing the specification that can extend our proposed debugging algorithm in such a way that can be applied to optimized arithmetic circuits while the techniques in [5][10] are not able to handle such designs, as will be discussed in Section V.

- Formally describing a wide range of arithmetic circuits including fast array dividers as well as sequential dividers by proposing functional bit-level and logical bit-level representations while the technique in [23] is just applicable to restoring and non-restoring dividers.

- Showing empirical results to prove that our proposed debugging technique enables us to verify and debug large industrial arithmetic circuits within practical time.

The rest of the paper is organized as follows: Section II presents the background on division arithmetic. In Section III basic representations are introduced. Section IV presents the proposed debugging technique for different types of division circuits. In Section V, we explain how to address various optimization issues. Section VI demonstrates experimental results and Section VII concludes the paper and presents the future works.

\section{DIVISION ARITHMETIC}

This section gives an overview of division algorithms taken into account in this paper, i.e., restoring, non-restoring, and SRT divisions. Combinational implementation of such divisions yield regularly structured fast array dividers, although division can hardly be implemented efficiently as pure combinational logic. However, before describing division algorithms, we need to define some terms that are frequently used in the rest of the paper.

Definition 1 (Dividend and Divisor): Let $A$ and $D$ be fixedpoint numbers. We say $D$ divides $A$, if there exists some fixedpoint number $K$ such that $A=K \times D$, where $A$ is a multiple of factor $D$. Then we call $A$ the dividend and $D$ the divisor.

Theorem 1 (Fixed-point Division): Let A and D be fixed-point numbers with $D \neq 0$. Then there exist unique fixed-point numbers $Q$ and $R$ such that $A=Q \times D+R$, where $0 \leqslant R<|D|$. Then we call $Q$ the quotient and $R$ the remainder.

The proof of the theorem can be found in [5].

For the rest of this paper let dividend $A=a_{0} \cdot a_{1} a_{2} \ldots a_{2 n}$, divisor $D=d_{0} . d_{1} d_{2} \ldots d_{n}$, quotient $Q=q_{0} . q_{1} q_{2} \ldots q_{n}$, and remainder $R=r_{0} . r_{1} r_{2} \ldots r_{n}$. Note that $q_{0}$ and $q_{n}$ are Most Significant Bit (MSB) and Least Significant Bit (LSB) of the quotient, respectively.

In most division algorithms, division is implemented by subtracting the divisor with a shifted value of dividend iteratively. In each iteration, the value of subtraction will be checked if it is negative or positive for determining the produced quotient. We call this value of subtraction in each iteration $i$, partial remainder $w_{i}$, that should be used in the next iteration. This process is very much the same as the subtraction process in ordinary paper and pencil division method that is subtracting the divisor from the shifted part of dividend in each iteration and producing the appropriate quotient and new dividend for the next iteration. The difference between division algorithms is in the production of partial remainder and selecting produced quotients. For example, in non-restoring dividers, after the subtraction of a shifted part of the dividend (or previous partial remainder) from divisor, the sign of the partial remainder will be checked and if it is negative, the divisor will be 
added to the partial remainder in the next iteration. But in restoring division algorithms, if the sign of partial remainder is negative, instead of an extra addition, the value of the shifted part of the dividend (or previous partial remainder) before subtraction will be propagated. In SRT division, with a special method of subtraction and comparing partial remainder with specific intervals, produced quotient(s) will be determined. In all above mentioned divisions, the addition/subtraction process, selecting produced quotient(s), and steering the partial remainder to the next iteration are the same, although they are implemented differently. Therefore a general form can be used to represent all above mentioned division algorithms as shown in Fig. 2.

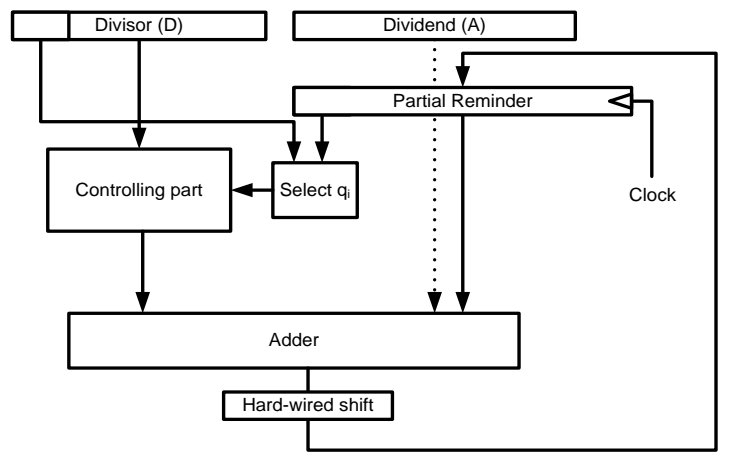

Figure 2. General form of various division algorithms

As can be seen in this figure, each division circuit contains an adder (or subtracter), a component for selecting and producing quotient(s) that is referred to as quotient selector function in the rest of the paper, and a component for steering the result of partial remainder and divisor to the adder that is called controlling function in this paper. In the rest of this section, this general form will be explained for three famous division algorithms, i.e. restoring, non-restoring, and SRT, although it can also be applied to any other division algorithms that use the division subtraction method. Without loss of generality, assume $a_{0}=d_{0}=0$ in the case of restoring division [9]. Division algorithms work by iteratively utilizing the following general equation:

$$
w_{i+1}=r . w_{i}-q_{i+1} d
$$

where, $d$ is the divisor, $r$ is the radix, $q_{i}$ is the $i^{\text {th }}$ quotient digit, and $w_{i}$ is the $i^{t h}$ partial remainder. Based on this we have the definition of Quotient Selector Function (QSF) as follows:

Definition 2 (Quotient Selector Function, QSF): the function that chooses the quotient based on comparison between the divisor and shifted partial remainder:

$$
q_{i+1}=Q S F\left(r, w_{i}, d\right)
$$

The implementations for the quotient digit selection part vary from design to design. The basic idea of QSF is to choose the value of the quotient digit, based on a comparison between the shifted partial remainder and the divisor. Finally, based on the above discussion, we define controlling function as follows:

Definition 3 (Controlling Function, CF): The function that identifies the inputs of the adder in division algorithms based on partial remainder, divisor, and the generated quotient from the previous iteration is denoted as $C F$.

In other words, $\mathrm{CF}$, as mentioned before, navigates the flow of partial remainder and divisor in each iteration of the division process based on the produced quotient in the previous iteration and partial remainder. Like QSF, the implementation of CFs depends on the type of the division algorithm. In the rest of this section we discuss QSF, CF, and more detail of structures for three division algorithms, i.e. restoring, non-restoring and SRT.

\section{Restoring Division}

Restoring division is the binary version of the traditional paper-and-pencil method. In the restoring division, if the remainder is greater than the divisor, the quotient bit is set to 1 and the divisor is subtracted from the remainder and then the partial remainder is shifted to the left. Otherwise, the remainder is simply shifted to the left. To compare the remainder with the divisor, a subtraction is needed, if the result is negative, the previous value is restored; that is why it is called restoring division. A general structure of a 7-by-4 restoring divider is shown in Fig. 3(a) which is based on Controlled Subtract (CS) cells. Each iteration performs (1) by one clock cycle where the quotient is computed based on the following equation in which QSF for a radix-2 restoring divider is taken into account.

$$
q_{i+1}=\operatorname{QSF}\left(w_{i}, d\right)= \begin{cases}1 & 2 \times w_{i}-d>0 \\ 0 & 2 \times w_{i}-d<0\end{cases}
$$

QSF for restoring divider is shown in Fig. 3(a). To check whether $2 \times w_{i}-d$ is greater/less than zero or not, two cases are considered. If $w_{i}$ is ' 1 ', definitely $2 \times \mathrm{W}_{\mathrm{i}}-d$ would be positive regardless of the value $d$. In the case of $w_{i}=0$, the generated quotient bit would be 1 if and only if the subtraction does not generate a carry out. This is the idea behind using a NOT gate and an OR gate in each row to generate corresponding quotient bits.

The partial remainder in $i^{\text {th }}$ iteration is determined by having the quotient generated in this iteration and the partial remainder of the previous iteration. The functionality of CF here is as follows:

$$
C F=C F\left(s_{i}, s_{i-1}, q_{i-1}\right)=\left\{\begin{array}{cc}
s_{i-1} & q_{i-1}=0 \\
s_{i} & q_{i-1}=1
\end{array}\right.
$$

where $s_{i}$ and $s_{(i-1)}$ are the generated sums in $i^{\text {th }}$ and $i-1^{\text {th }}$ iterations; $\mathrm{CF}$ for restoring divider that is a multiplexer as shown in Fig. 3(b).

\section{Non-Restoring Division}

Although there are practical implementations of restoring division in hardware, they are actually never used because of the restoring step which can cause the division process to require up to $2 n$ iterations in the worst case. The restoring step can be avoided through an improved method called non-restoring division which keeps processing with a negative result. This means that the partial remainder can be either positive or negative and therefore the operation to be performed can be either subtraction or addition. A general structure of a non-restoring divider is illustrated in Fig. 3(c). This scheme consists of carry-propagate adders through multiple logic cells. As shown in Fig. 3(d), each Controlled Add/Subtract (CAS) cell consists of a full adder and an exclusive-or gate which controls the input of the next row, i.e. CF.

The previously generated quotient bit determines whether an addition or subtraction is to be performed. Subtraction is performed in 2's complement by using 1's complement of the divisor and forcing a carry into the rightmost cell. The quotient 
bit $q_{i}$ depends on the sign of the partial remainder which is determined from the carry-out of the leftmost cell. Therefore if the partial remainder is less than zero, $q_{i}=0$ and if the partial remainder is greater than zero, $q_{i}=1$. Therefore the quotient selector function for non-restoring algorithm is defined as follows:

$$
q_{i+1}=\operatorname{QSF}\left(w_{i}, d\right)= \begin{cases}1 & w_{i}>0 \\ 0 & w_{i}<0\end{cases}
$$

Hence, in non-restoring division, $\mathrm{CF}$ can be expressed by:

$$
C F=C F\left(d, q_{i-1}\right)=q_{i-1} \oplus d
$$

where $q_{i-1}$ is the generated quotient in the previous iteration and $d$ is the divisor.

\section{SRT Division}

The most efficient method to perform division is the SRT (Sweeney, Robertson, and Tocher) method which allows 0 to be a quotient digit for which no addition or subtraction is performed [9]. In other words, compared to the quotient digit set $\{-1,1\}$ used in radix-2 non-restoring division, the set for radix2 SRT becomes $\{-1,0,1\}$. Fig. 3(e) shows the implementation of radix-2 SRT divider. The advantage of the SRT algorithm is that, we compare the remainder with 0.5 instead of the dividend. Its drawback is the non-unique form of final quotient because of making use of redundant quotient-set digit set, namely $\{-1,0,1\}$. The binary representation of the mentioned digit set needs two bits for each digit. So, a final conversion on the generated quotient is needed. The flow of data between adders is controlled by a controlling function (see CF in Fig. 3(e)). The quotient selector function (QSF) for radix-2 SRT algorithm is expressed as follows:

$$
q_{i}=\left(q i_{-} p o s, q i_{-} n e g\right)=Q S F\left(w_{i-1}, d\right)=\left\{\begin{array}{rr}
1,0 & 2 w_{i-1} \geq \frac{1}{2} \\
0,0 & -\frac{1}{2} \leq 2 w_{i-1} \leq \frac{1}{2} \\
0,1 & 2 w_{i-1} \leq-\frac{1}{2}
\end{array}\right.
$$

A binary fraction in 2's complement representation is greater than or equal to 0.5 if and only if it starts with $(0.1)_{b}$ and is less than or equal to -0.5 if and only if it starts with $(1.0)_{b}$. Therefore, in each iteration, only 2 bits of partial remainder are examined and there is no need to compare the whole partial remainder with the dividend. In radix-2 SRT division $\mathrm{CF}$ for each cell is similar to that of non-restoring division:

$$
C F=C F\left(d, s_{i-1}, q_{i-1}\right)= \begin{cases}0 & q_{(i-1) \_n e g}=0 \\ d \oplus q_{(i-1) \_p o s} & q_{(i-1) \_n e g}=1\end{cases}
$$

where $d$ is the divisor and $q_{i}=\left\{q_{i \_p o s}, q_{i \_n e g}\right\}$.

The idea of the SRT division algorithm can easily be generalized to higher radixes [9]. The block diagram of radix-r divider hardware is shown in Fig. 4. Note that this radix-r divider is similar to radix-2 divider in Fig. 3(e), except it consists of larger general multiple dividend generation circuit and more quotient digit selection logic.

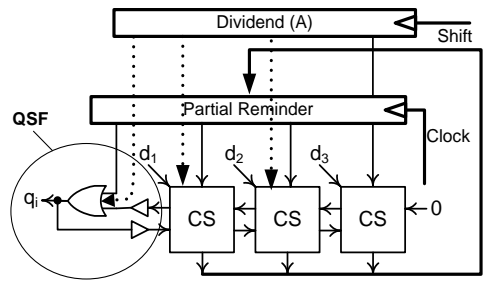

(a)

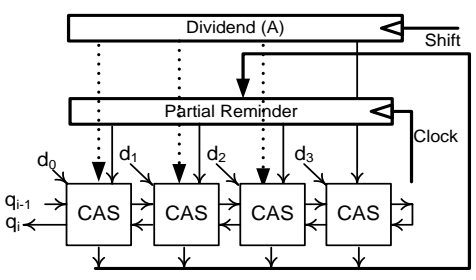

(c)

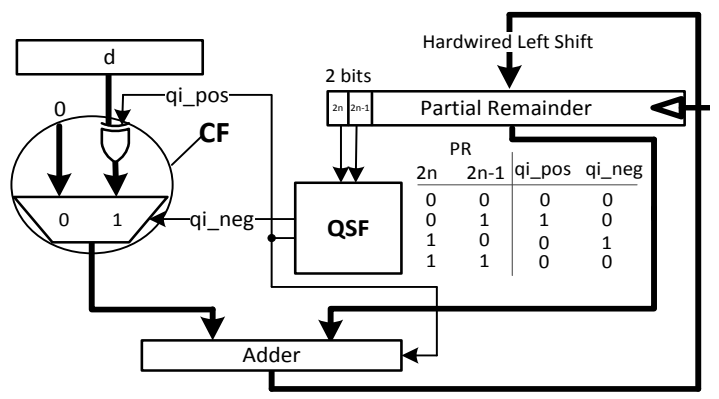

(e)

Figure 3. (a) General structure for 7-by-4 restoring divider, (b) Controlled Subtract (CS) component, (c) General structure for 7-by-4 non-restoring dividers, (d) Controlled Add/Subtract (CAS) component, (e) Radix-2 SRT Divider

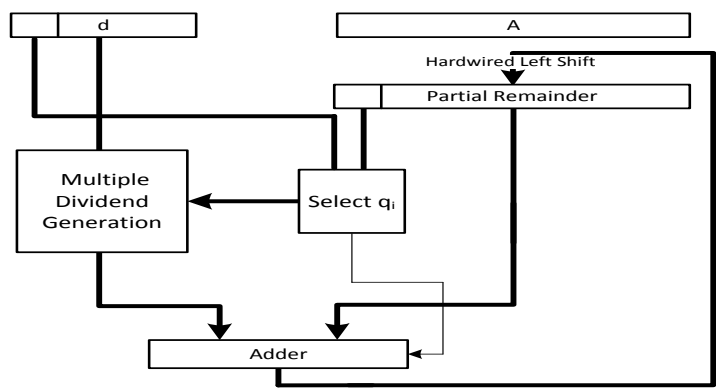

Figure 4. Structure of a radix-r SRT divider

\section{Fast Array Dividers}

As mentioned before, there exist methods for implementing division as pure combinational logic. In this subsection we give an overview about such methods that are called fast array dividers or cellular array dividers.

As discussed before, with sequential division all intermediate results must be stored in order to prepare for the next step. Although the hardware required for designing iterative circuits is simple, it is also slow, and thus, leads to relatively long execution time. In order to alleviate this problem, a basic idea is to replace successive write and shift operations with a combinational circuit that forwards intermediate results without storing them. For this purpose, an array of cells is used 
where each step of the sequential process is executed by a separate row of cells. Consequently, a matrix of $n$ rows, where each row consists of $n$ cells, must be implemented for performing $n$-bit division as shown in Fig. 5. This figure illustrates a combinational array structure for a restoring divider which is essentially a time-frame expansion of the circuit in Fig. 3(a).

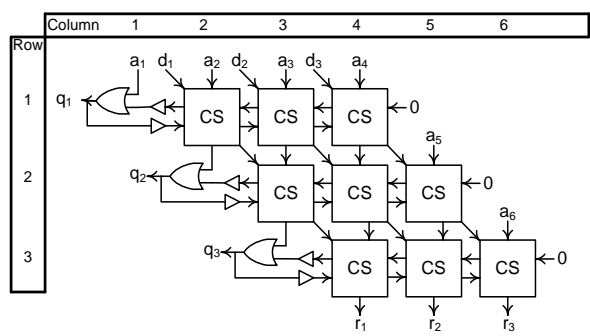

Figure 5. Restoring array divider

\section{FUNCTIONAL BIT LEVEL REPRESENTATION}

Our debugging technique is based on equivalence checking between a high level specification and a gate-level implementation. However, the high-level specification is very general and needs to be reduced to a lower level of abstraction. This reduction process must be performed based on the information obtained from the implementation during its analysis. This problem raises two interesting questions: 1) how such information obtained from the implementation can be used for generating lower level of abstraction of the specification, and 2) what sort of information from the implementation can help us to reduce the high-level specification as much as possible. To answer the questions we propose Functional Bit Level Adder (FBLA) that is a high-level abstract model of an arithmetic circuit in the form that its repetitive components in the implementation are functionally separated from each other as much as possible. As discussed in the previous section, division circuits, like many other arithmetic circuits, are constructed from repetitive subcomponents such as HAs, FAs, and repetitive cones. So identifying such logics in the implementation can help us to gradually update and construct FBLA. As an example, Fig. 6 represents an FBLA generated by an implementation of a divider. Based on the structures of dividers discussed in Section II (both combinational and sequential ones), FBLA in this figure is an addition network that contains three main components CFs, QSFs, and Adders. Based on (4), (6), and (8), $\mathrm{CFs}$ for non-restoring, restoring, and SRT dividers are different as can be seen in Fig. 6(a), (b), and (c), respectively. As can be seen, the general form of the bit level adder representation in Fig. 6(d) supports all types of divisions.

Although various optimizations in the implementation which are hidden from the high level specification, can be applied, limited forms of FBLAs are sufficient to support such optimizations as the synthesis results of various divider circuits show. For example, in addition to different division algorithms, i.e. restoring, non-restoring, and SRT, adder blocks can be implemented in many different ways such as carry lookahead mechanism, instead of carry ripple one. In such cases, FBLA is easily updated according to the optimization by merging some of its blocks. As the process of formal verification and debugging is the same for different structures of FBLAs, we explain our debugging technique according to the FBLA shown in Fig. 6.

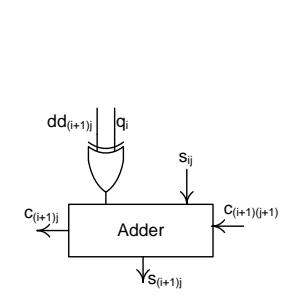

(a)

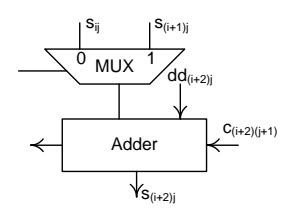

(b)

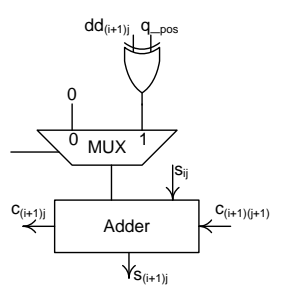

(c)

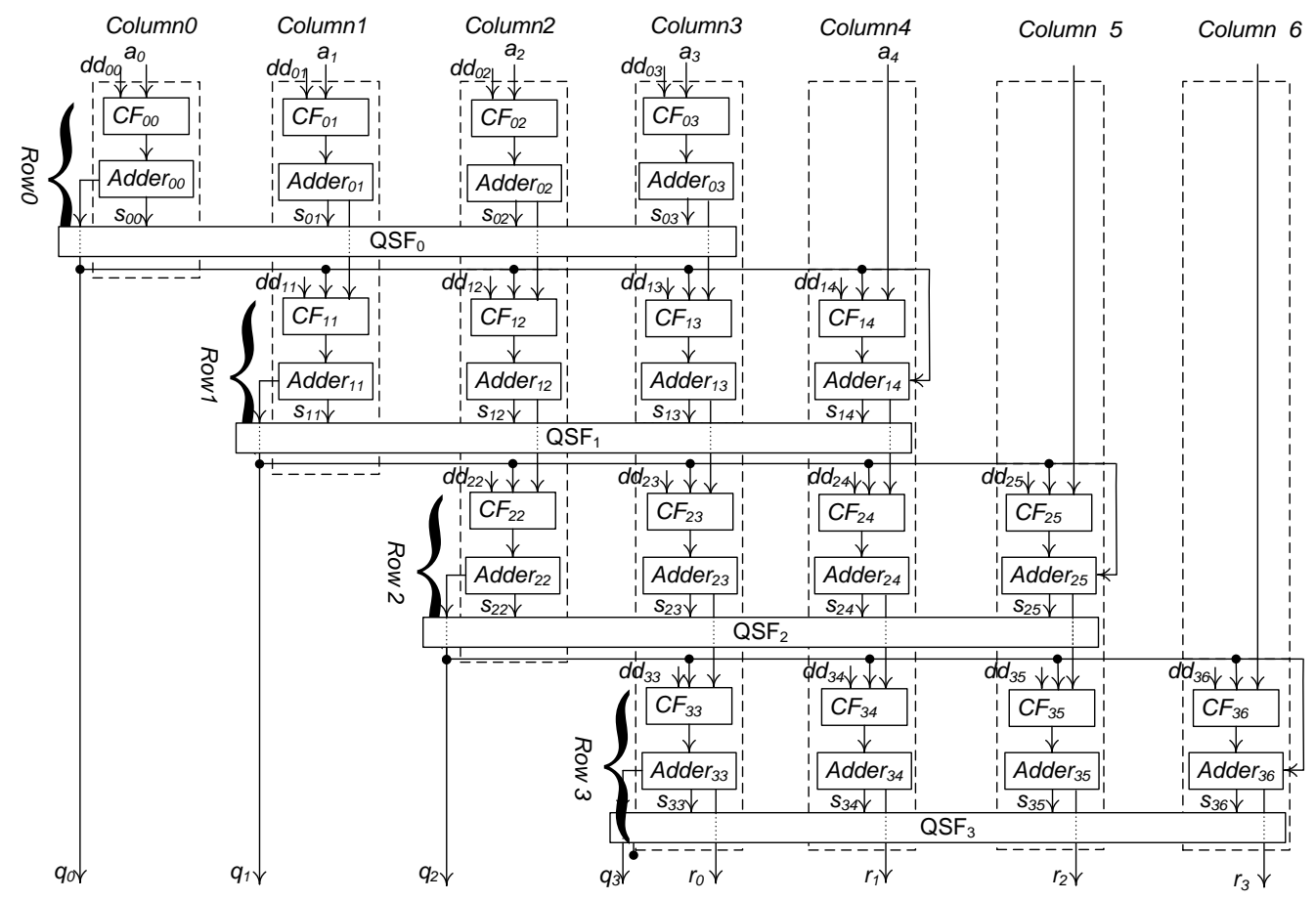

(d)

Figure 6. The main components in FBLA representation (a) for non-restoring dividers, (b) for restoring dividers, (c) for SRT radix-2 dividers, and (d) FBLA representation for 7-by-4 division, where $q_{i}$ is the carry result of the leftmost Adder in Row $i$ and $r_{j-n+1}$ is the sum result of the last Adder in Column $j$

In FBLA, like array dividers presented in subsection II4 , each iteration of the division algorithm is labeled by a row and each column represents one bit of addition. As shown in Fig 6, each Row contains one QSF, several one bit adders and CFs. In Row $i$ and Column $j$ we have one adder and one CF that forms $\mathrm{Cell}_{i j}$. The output of the adder in $C e l l_{i j}$ is shown as $s_{i j}$. The inputs of each $C e l l_{i j}$ come from a previously-generated quotient by $\mathrm{QSF}_{\mathrm{i}-1}, q_{i-1}$, output of the 
previous adder in the same column, $s_{(i-1) j}$, and the divisor. As the index of divisor differs in different division algorithms, we used term $d d_{i j}$ for the index of the input divisor in each cell that depends on the type of the division algorithm. For restoring division algorithm $d d_{i j}=j-i+1$ and for non-restoring and SRT division algorithms $d d_{i j}=j-i$. For example, Fig. 6(d) illustrates FBLA of a 7-by-4 division function where 4-bit $D$ divides 7-bit $A$. Although we ensure that cascaded CFs, Adders, and QSFs exist in each column, we do not know how the carries are propagated through the rows. That is why there is no connection between columns in Fig. 6(d). As a simple example, in a non-restoring divider the carry propagation function is determined as follows when a ripple carry structure is used:

$$
\mathrm{CPF}_{\mathrm{ij}}=\mathrm{CPF}_{\mathrm{i}} \mathrm{S}_{(\mathrm{i}-1) \mathrm{j}}+\mathrm{CL}_{\mathrm{ij}} \mathrm{S}_{(\mathrm{i}-1) \mathrm{j}}+\mathrm{CL}_{\mathrm{ij}} \mathrm{CPF}_{\mathrm{i}}
$$

Such connections and types of CFs and QSFs are identified during the process of the gate level implementation, as will be explained in Section IV. Before presenting the proposed approach, we mention a theorem that is used in the proposed debugging algorithm later.

Theorem 2: In an array divider, there is no way to optimize $C L$ logics of adjacent rows $i$ and $i+1$. In other words, no optimization technique is able to combine $C L_{i j}$ and $C L_{(i+1) j}$ in Fig. 7.

Proof: Suppose two respective CLs in Row $i$ and $i+1$ in Column $j$ are combined together producing the Quotient $i$ and $i+1$ together. According to Definition 3 in Section II, for array dividers each $\mathrm{CF}$ contributes to calculate the current quotient in each row based on produced quotient from the previous row. Therefore Quotient $i+1$ must be generated based on Quotient $i$. However, this is against our first assumption through which all two quotients were produced together at the same time. Note that the generated quotients are also the primary output of the divider and cannot be hidden. Thus, each CL must be independent from the other adjacent CLs in each column.

\section{PROPOSED DEBUGGING TECHNIQUE}

In this section, we present a debugging technique for gate-level arithmetic circuits. Assume a polynomial expression as a high level specification (spec) and a gate-level implementation (impl) is given. We say that the gate-level implementation is correct iff (if and only if) it is functionally equivalent to the given specification. Otherwise, the implementation is buggy. Please note that the two models are functionally equivalent if they generate the same outputs given the same inputs. Using high level specification although we can localize all bugs, some of them can be automatically corrected.

We make use of a reverse engineering mechanism to describe impl at a higher level of abstraction where arithmetic operations at bit level are defined. To represent such information, we have proposed Logical Bit Level Adder (LBLA) representation that contains four main components, i.e. sum generation logic (SGL), carry propagation logic (CPL), controlling logic (CL), and quotient selector logic (QSL). Fig. 7 shows LBLA of a 7-by- 4 restoring divider $\left(a_{0}=d_{0}=0\right)$ that corresponds to FBLA in Fig. 6(d) where Adders are partitioned into two parts, i.e., SGL and CPL, as you can see in Fig. 7. In addition, QSF in Fig. 8(d) corresponds to QSL in Fig. 7 which is carry propagation logic for the most significant bit of the partial remainder for non-restoring dividers.

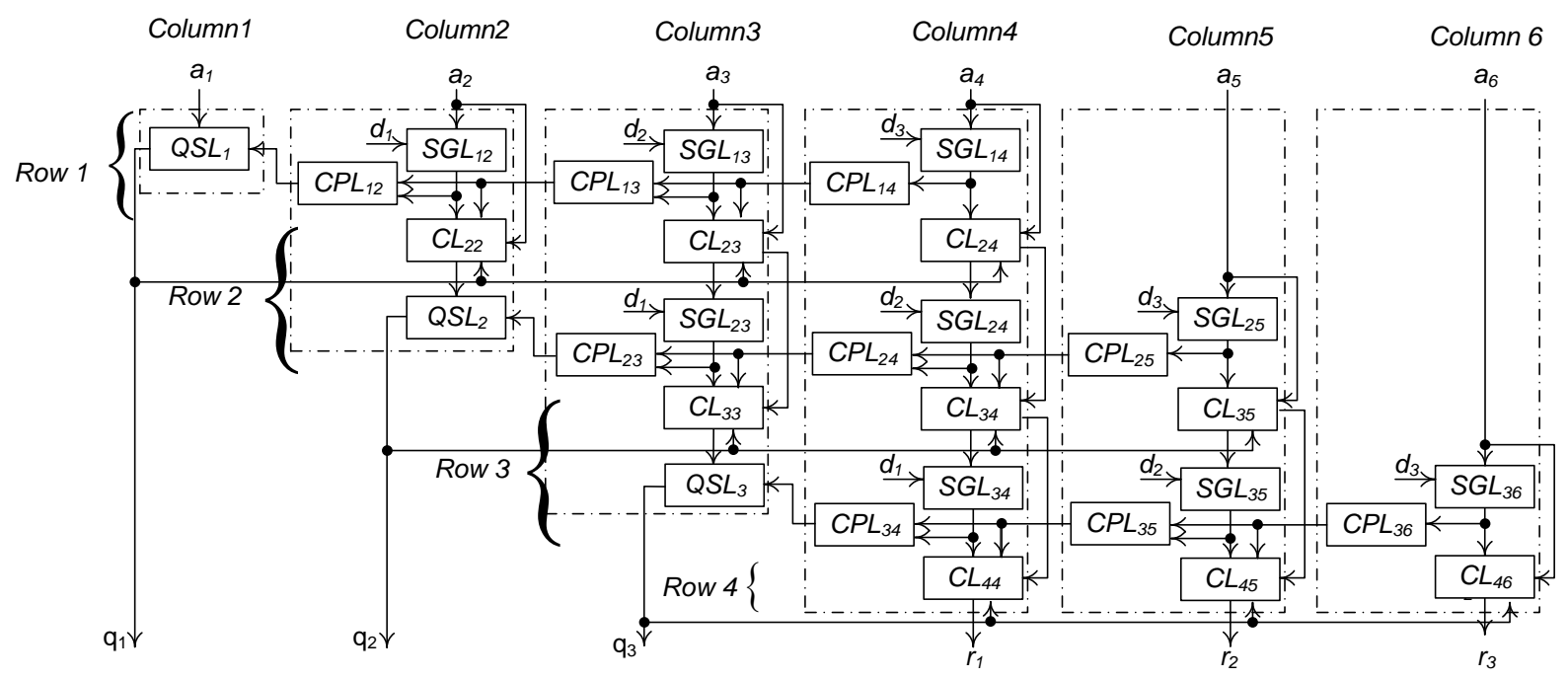

Figure 7. LBLA representation for 7-by-4 restoring divider containing four main components: SGL (Sum Generation Logic), CPL (Carry Propagation Logic), CL (Controlling Logic), and QSL (Quotient Selector Logic)

The pseudo-code of the proposed method is shown in Algorithm 1. The algorithm starts by a preprocessing phase in which the initialization of FBLA and time frame expansion of implementation for sequential circuits will be done (line 1 of Algorithm 1). Our proposed debugging technique consists of four main phases that will be explained in more details in the following subsections:
1. SGL extraction/verification phase in which XORs are extracted and then mapped to HAs and FAs and finally verified (line 2). If SGL extraction fails, cone extraction/verification phase would be done where repetitive cones will be extracted from the implementation (line 4). 
2. CPL extraction/verification phase in which the functionality of carry propagation logics are determined and then verified (line 7).

3. QSL extraction/verification phase in which the functionality of quotient selector logic is determined and then verified (line 8).

4. CL extraction/verification phase in which the functionality of controlling logics is determined and then verified (line 9).

After SGL/cone extraction, the algorithm applies a preprocessing analysis to identify the type of the division algorithm according to the functionality of CLs and QSLs in the first row of the implementation (line 5).

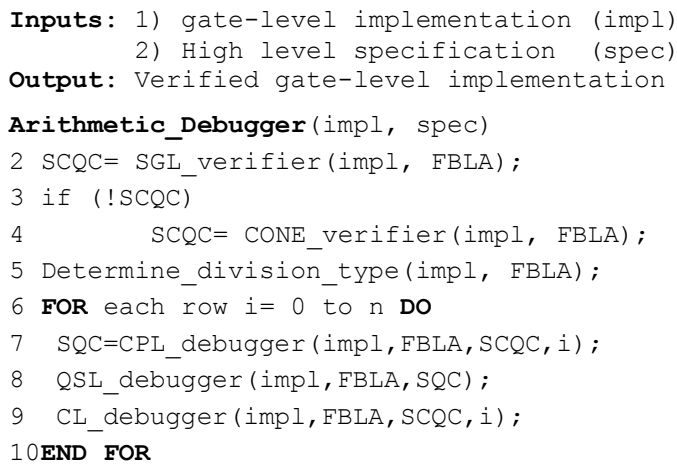

Algorithm 1. Proposed debugging algorithm

\section{Sum Generation Logic (SGL) Verification}

In this section we explain our algorithm to verify SGL as illustrated in Algorithm 2. First of all, a column based XOR-extraction mechanism is employed to extract XORs (line 2 of Algorithm 2) and then map them to half-adders (HAs) and full-adders (FAs). If this process fails, according to non-XOR gates for SGLs or any optimization, the function returns zero showing that cone verifier needs to be applied. Otherwise, based on the extracted components (i.e., HAs and FAs) the FBLA is updated. During this updating process, adders (i.e., FAs and HAs) will be determined in the FBLA. After that, those primary inputs that are connected to the extracted adders identify the row of each adder (line 5 in Algorithm 2). Note that those inputs of adders driven by carry propagation logics or controlling logics are referred to as suspected CPL, QSL, or CL (SCQC) nodes in the rest of the paper. We make use of such nodes (SCQC in line 6) to verify CPLs and CLs in the next phases. It can be observed that CPF and CF can be separated from each other whenever we have adders and SCQC nodes. Hence, for each implementation in which HAs and FAs can be extracted, high-level specification can be reduced to the general form of FBLA in Fig. 6. Therefore, during the SGL extraction, FBLA should be updated too (lines 2-3 in Algorithm 2).

As FBLA is being updated, LBLA would be constructed too. One important point to be noted here is that the XOR extraction mechanism in [11] cannot be used because there might be CLs between SGLs as you can see in the example of Fig. 7. To overcome this problem, the algorithm starts from $a_{j}$ (in Column $j$ ) by looking for XORs until non-XOR gates are reached. Such non-XOR gates that are part of CLs are bypassed until an XOR gate is reached. If such an XOR gate is not a part of SGLs in Column $j-1$, it belongs to an
SGL in Column $j$. This process continues until reaching primary outputs in Column $j$. The following observation shows that the non-XOR gates detected in this process are definitely part of CLs.

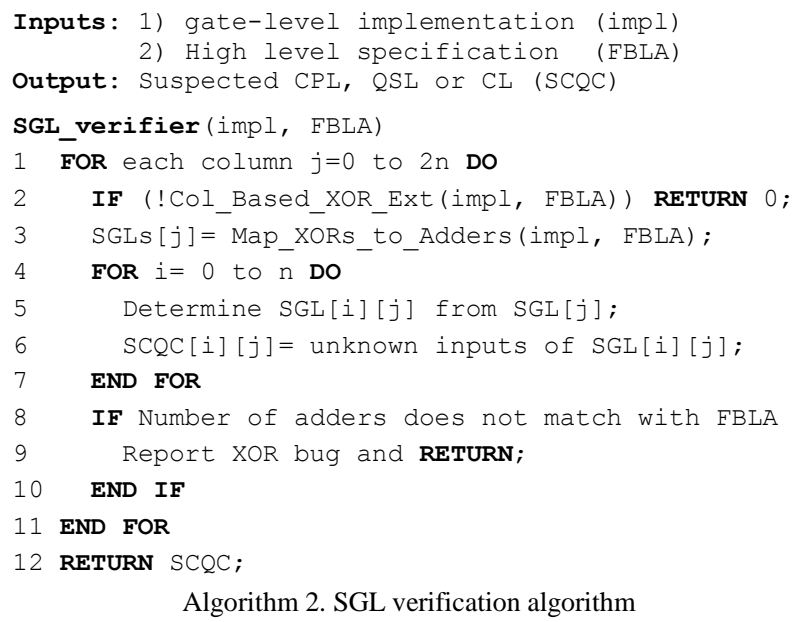

Based on Theorem 2, it can be concluded that in a gate level implementation of dividers with XOR-based HAs and FAs, non-XOR gates between SGLs in a given Column $j$ are parts of CLs in Column $j$. To clarify this observation, let us consider a part of the gate-level restoring divider in Fig. 8. Two grey colored cascaded XORs indicating FAs in Column $j$ are labeled as $\mathrm{SGL}_{1 \mathrm{j}}$ and $\mathrm{SGL}_{2 \mathrm{j}}$. While the dotted gates in Column $j$ show those gates of $\mathrm{CL}_{2 \mathrm{j}}$ that are detected during SGL extraction. In order to verify SGL, if the number of XORs in Column $j$ is less than that of FBLA the algorithm reports that XORs are buggy and exits (lines 8-10 of Algorithm 2). Note that we make use of the fact that the number of XORs in a column can be increased by parallelism or similar techniques, but no logic optimization technique can reduce the minimum number of required XORs (e.g.) [5 Th. 1]. The other assumption that should be considered here is that all the design is synthesized with the same rule and we have XOR-based HAs and FAs all over the design except the borders.

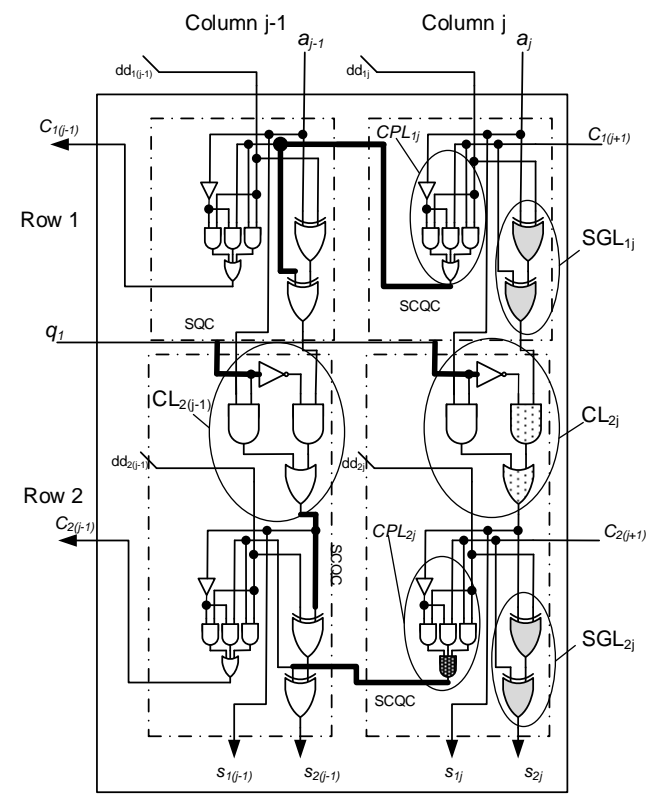

Figure 8. Part of a buggy gate-level implementation (a restoring divider) 
In FBLA the location and functionality of each component will be determined while processing the implementation. If the process of SGL extraction succeeds, i.e., no cone verifier is needed, then the location of adders (i.e., FAs and HAs) in the FBLA is fixed. For example Fig. 9(a) shows the updated FBLA for the implementation presented in Fig. 8 after SGL extraction. As it can be seen, based on the SGL extraction, the location of adders and controlling functions (i.e., CFs) are determined in the FBLA.

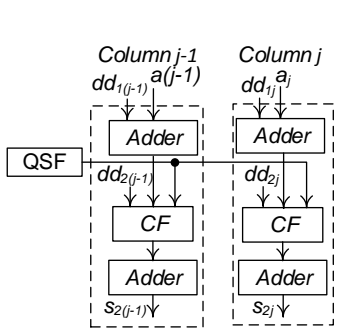

(a)

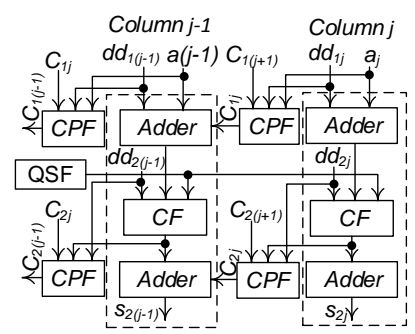

(b)
Figure 9. FBLA updating process, (a) after SGL extraction, (b) after CPL extraction

\section{CPLs extraction and debugging}

As mentioned before, during the SGL extraction phase, there exist XORs with unknown inputs (see $S C Q C$ in Fig. 8) that are related to CPLs (or CLs). Algorithm 3 illustrates how to extract and then debug CPLs. As carry signals are propagated through rows, this extraction phase starts processing the row $i$ from Column $n+1$ down to $i$ (line 1 in Algorithm 3). To extract CPLs, a backtracking process by starting from a SCQC node is done (line 2). This process continues until reaching a primary input, SGL, previously specified CL or CPL or QSL (line 3). Logics extracted are considered as CPL (line 4). If, however, a known node from previous columns is reached the extracted logics are CLs instead of CPLs. Some inputs of such CLs are coming from QSL which are unknown at this phase (lines 7-9). We refer to these unknown nodes as suspected QSL, or CL (SQC) nodes in the rest of the paper. Note that QSL verification that will be explained in the next subsection is based on SQC nodes.

In FBLA, the functionality of each input node according to the available inputs, the inputs can be primary inputs or the known nodes from previous step(s), should exist. However, as we do not have any information about the inputs of CPLs, CLs, or QSLs, the algorithm postpones updating the functionality of CPF, CF, and QSF until finding the known inputs from CPL, CL, and QSL. During the back tracking process, in each iteration, after finding the known inputs, FBLA will be updated according to known inputs and the extracted logic will be used to update LBLA. After equivalence checking between FBLA and LBLA, the corresponding unknown node will change to known node and the algorithm continues with another unknown node (line 10). For example for the implementation in Fig. 8, during processing Column $j-1$, the SCQC highlighted in Row 1 is backtracked until reaching $a_{j}, c_{l(j+1)}$, or $d d_{l j}$. Fig. 9(b) shows the updated FBLA of the implementation in Fig. 8 after extracting CPLs. In order to verify the functionality of the extracted logic, equivalence checking between the extracted $\mathrm{CPL}_{1 \mathrm{j}}=\bar{a}_{j} \cdot d d_{1 j}+\bar{a}_{j} \cdot c_{1(j+1)}+c_{1(j+1)} \cdot d d_{1 j}$ and the expected $\mathrm{CPF}_{1 \mathrm{j}}$ from FBLA is carried out. As $\mathrm{CPL}_{1 \mathrm{j}}=\mathrm{CPF}_{1 \mathrm{j}}$ based on
(9) we conclude that $\mathrm{CPL}_{1 \mathrm{j}}$ is properly implemented at the gate level.

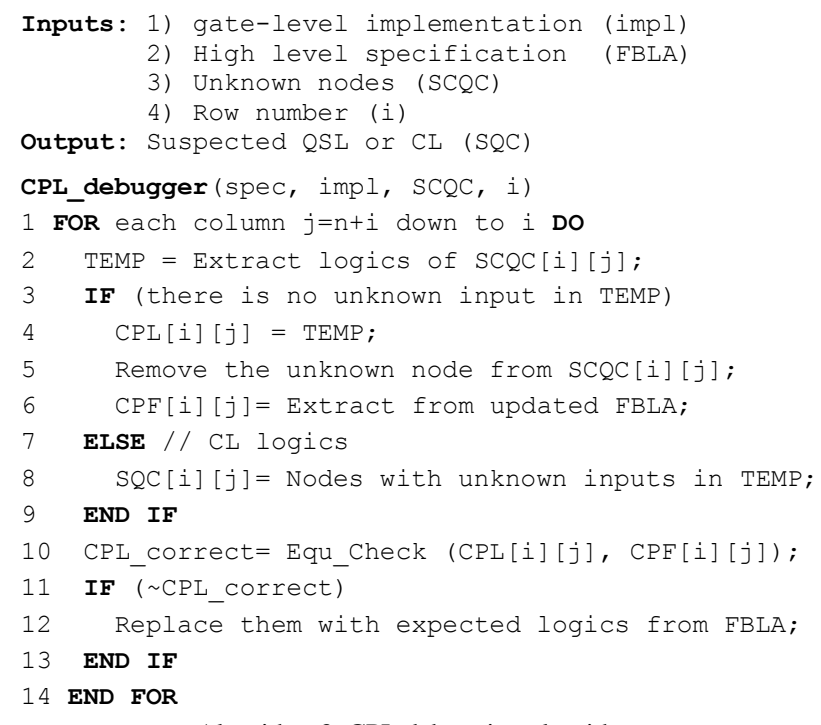

Algorithm 3. CPL debugging algorithm

As discussed above, in order to verify the extracted CPLs, their functionalities are compared with the expected ones extracted from FBLA using conventional equivalence checkers (line 10). If, however, they are not equivalent, the extracted CPLs are replaced with the expected ones to automatically correct them (lines 11-13). In Fig. 8, during processing Column $j-1$, the $S C Q C$ highlighted in Row 2 is backtracked until reaching $C L_{2 j}, c_{2(j+1)}$, or $d d_{2 j}$. In order to verify its functionality, equivalence checking between the extracted logic, i.e., $C P L_{2 j}=\overline{C L}_{2 j} \cdot d d_{2 j} \cdot c_{2(j+1)}$, and the expected logic, i.e., $C P F_{2 j}=$ $\overline{C L}_{2 j} \cdot d d_{2 j}+\overline{C L}_{2 j} \cdot c_{2(j+1)}+c_{2(j+1)} \cdot d d_{2 j}$, from FBLA is performed. It is obvious that they are not equivalent and therefore to correct the bug, the debugging technique substitutes $\mathrm{CPL}_{2 \mathrm{j}}$ with the expected one, i.e., $\mathrm{CPF}_{2 \mathrm{j}}$. Please note that while extracting CPL, the type of carry propagation logics is determined.

\section{QSLs extraction and debugging}

Algorithm 4 illustrates QSL debugging algorithm. In order to extract and then debug QSL, we continue backtracking from $S Q C$ nodes until reaching primary inputs (line 1 of Algorithm 4). On the other hand, its main functionality is extracted from FBLA based on (3), (5), or (7). In order to verify the extracted QSL, its functionality is compared with an expected one extracted from FBLA using conventional equivalence checkers (line 3). If, however, they are not equivalent, the extracted QSL is replaced with the expected one to automatically correct it (lines 4-6). For example, Fig. 10 depicts QSL of restoring divider shown in Fig. 8 that should be compared with (3).

It should be noted that the type of the division algorithm specifies which equation, i.e., (3), (5), or (7), should be used to determine the functionality of QSL (line 2). If the type of division algorithm is restoring or non-restoring, a single output QSL should be extracted so that its equivalence with (3) or (5) needs to be checked. In the case of radix-2 SRT division, a two output QSL is extracted so that its functionality should be compared with (7). For example, Fig. 11 
shows a part of the gate-level implementation of a radix-2 SRT division algorithm. As can be seen in the figure, the quotient selector logic (QSL) is more complex than what represented in Fig. 10. Such complex QSL has two outputs, i.e., q_pos and q_neg, which are $S Q C$.

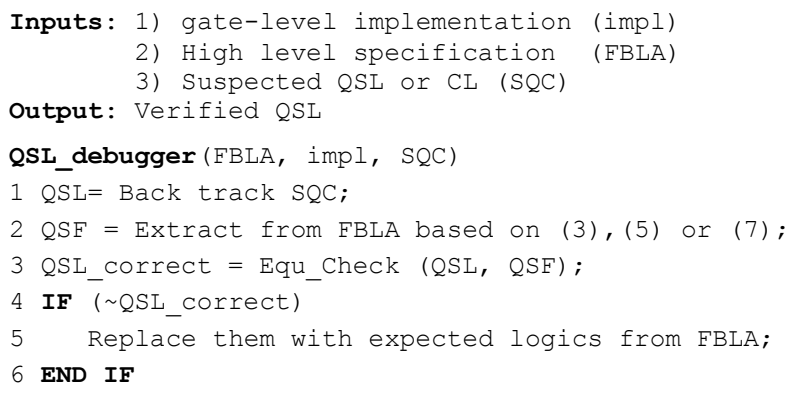

Algorithm 4. QSL debugging algorithm

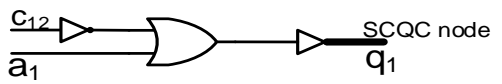

Figure 10. Gate level implementation of quotient selector logic (QSL) for restoring divider

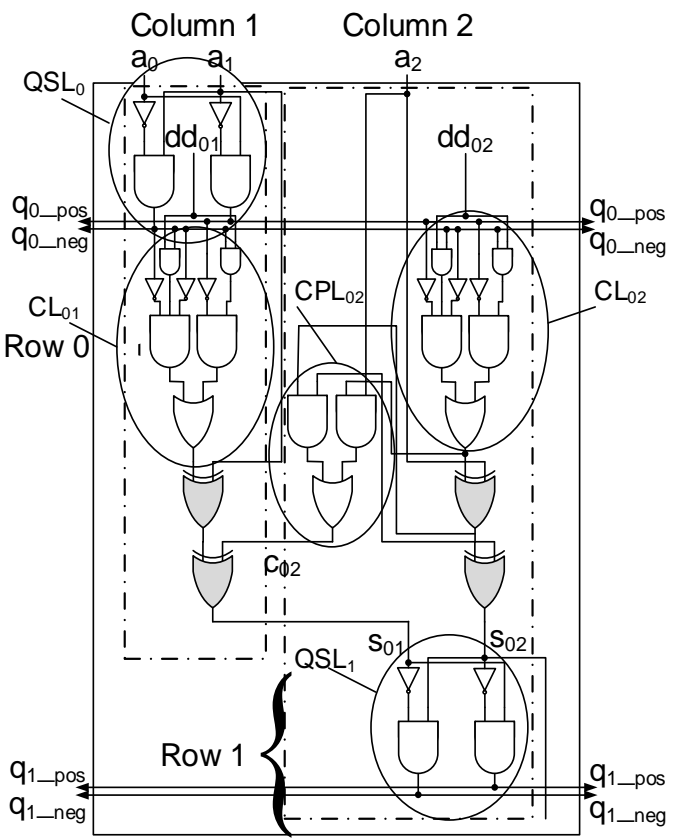

Figure 11. Part of a gate-level implementation of radix-2 SRT divider

It is worth noting that the proposed QSL debugging mechanism is also applicable in the case of speeding up the remainder update stage in the implementation where the design makes use of a sum and carry representation for the remainder; the remainder actually never exists as a single signal vector in the design, but is represented implicitly as the sum of two vectors, i.e. sum+carry. This avoids the need for a carry propagation chain in the remainder update. However, quotient selection will then no longer have the luxury of being aware of the pattern of the top bits of the true remainder, and will rely instead on some approximation of them. This approximation, and the way the approximation is used is often the most crucial part of the divider implementation, and the hardest to get right. For example, the Pentium FDIV bug in 1995 which is discussed extensively in the academic literature, was caused by an incorrect optimization in the quotient selection mechanism, causing the remainder to stray from the convergence bound $|R|<D$ of the algorithm.

\section{CLs extraction and debugging}

Algorithm 5 illustrates how to extract and then debug CLs from the remaining SCQC nodes. Like carry signals, this extraction phase starts processing the row $i$ from Column $n+i$ down to $i$ (line 1 of Algorithm 5). To extract CLs, a backtracking process by starting from a $S C Q C$ is done. This process continues until reaching a primary input, SGL, previously specified CL or CPL or QSL. As CPLs have been extracted in the previous phase, all logic extracted in this phase are considered as CLs (line 2). On the other hand, the expected controlling function (CF) is extracted from updated FBLA as well as CPL extraction phase (line 4). Finally equivalence checking between $\mathrm{CL}$ and $\mathrm{CF}$ is done (line 5).

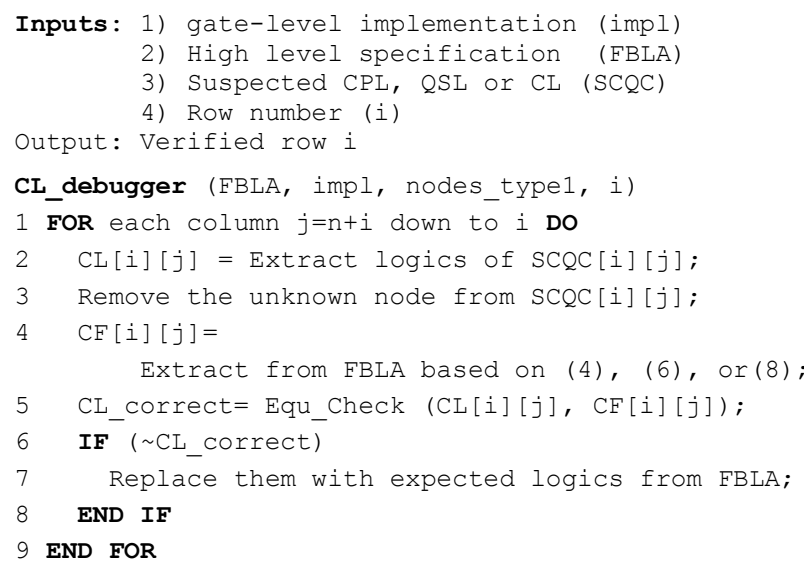

Algorithm 5. CL debugging algorithm

\section{Caveats and considerations}

There are some caveats and considerations regarding to the proposed algorithm. The first issue is the limitation of the proposed algorithm to update the FBLA when the structure of the implementation is too complicated. Such structures can be determined when in the initial stage of CONE_verifier function the algorithm cannot find the functionality of the cone. In this case the algorithm stops showing it is not able to update FBLA. Not that for the division circuits that are synthesized from truth tables, if the design is small and in the synthesis process, HAs and FAs are not detectable, then it is possible for the algorithm to extract the functionality of FBLA components by combining the subcomponents of FBLA in Fig. 6. However, if the design is not small, then the algorithm might not be able to construct the FBLA in its initial stage and it terminates. It is worth mentioning that the synthesis process for a large design with truth table is time consuming and seems not to be worthwhile.

The other issue that should be considered in the proposed verification and debugging method is the ability of the proposed algorithm to correct only the CPL, QSL, and CL but not the SGL. The reason is that, the debugging process can be done only when the inputs of the subcomponent are known inputs. However, while extracting SGLs, the verification process only is done by counting the number of adders in each row and column and comparing this with the 
expected one from specification. Therefore in this step (i.e., SGL extraction) no debugging process happens.

\section{SYNTHESIS OPTIMIZATION ISSUES}

According to our explanation in the previous section, it is obvious that FBLA has been introduced as a high level representation of those division circuits in which SGLs, CPLs and CLs are completely separated from each other. There are some optimization techniques that combine sum generation logics with carry propagation logics or controlling logics as shown in Fig. 12. In such circuits XOR chain of SGLs does not exist because XORs are combined with CPLs or CLs. All the mentioned structure variation and optimization process can be categorized into two parts, 1) implementations in which XOR chain of SGLs exists, and 2) implementations in which XORs are combined with CPLs or CLs.

In all cases in which XOR-based SGLs can be extracted, the general form of FBLA in Fig. 6 can be used. For example in SRT dividers, for accelerating the division process, generating needed carries for the next phase of quotient generation will be done by fast carry generation processes like carry-save or carry look ahead. However, SGLs are XORs. Fig. 13 shows two CPL extractions while using CLA, Fig. 13(a), and carry-save adder, Fig. 13(b) that are shown by grey colors. These gates are extracted according to Algorithm 1 from SCQC nodes. Note that in FBLA, the functionality of the extracted logics in such cases should be updated while extracting CPL. Fig. 13(c) and (d) demonstrate the CPF in FBLA corresponding to Adder $_{\alpha}$ that is updated according to the proposed algorithm. As it can be seen, for CLA the CPF (Fig. 13(c)) gets five inputs coming from previous steps that shows the process of updating FBLA is flexible w.r.t. the back tracking process in the implementation.

There are two forms in which XOR chains of SGLs are implemented with non-XOR gates. In one case, due to some constraints on cell library of synthesis tool, XORs are implemented by other gates. In such cases first of all non-XOR gates should be converted to XOR gates and after that, the proposed debugging technique can be applied. In some other cases, the functionality of SGLs is combined with other components and SGL extraction cannot be applied. In such cases, the algorithm tries to find repetitive cones all over the implementation by analyzing the fan-outs. It is obvious that FBLA of Fig. 6 cannot be used and we need more abstract FBLA. Algorithm 6 demonstrates the CONE_verifier function. This algorithm tries to find repetitive similar cones all over the design using a trial and error search method. In each iteration an unlabeled fan-out will be backtracked until reaching a labeled fan-out or a primary input (lines 3-4 in Algorithm 6). The extracted logics in this phase will be considered as a cone that will be used to update FBLA. If FBLA can be updated, this cone will be searched all over the design for update FBLA as much as possible (line 5). If we find such structures in the design, $S C Q C$, will be unknown inputs of the cones (lines 6-8). The initial form of FBLA is as Fig. 6. By extracting the cone (and extracted primary inputs of the cone), the algorithm tries to merge separated components in Fig. 6 to find a more abstract possible model for FBLA. If it fails, the algorithm labels the fan-out and tries to do the same with another unlabeled fan- out. The parameter $i$ determines that fan-out searching process starts from the first row in column $n$.

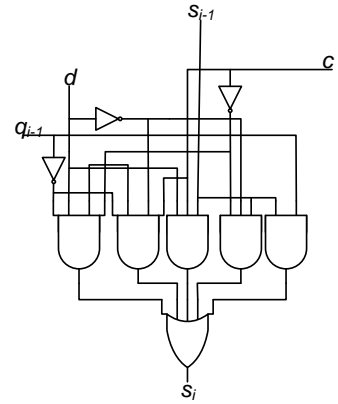

(a)

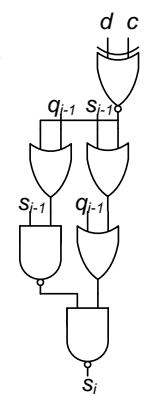

(b)

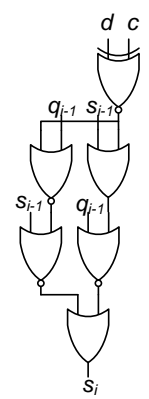

(c)
Figure 12. Three possible optimizations for restoring divider (a) available in text books (b) synthesized by ASIC synthesis tool (c) synthesized by FPGA synthesis tool

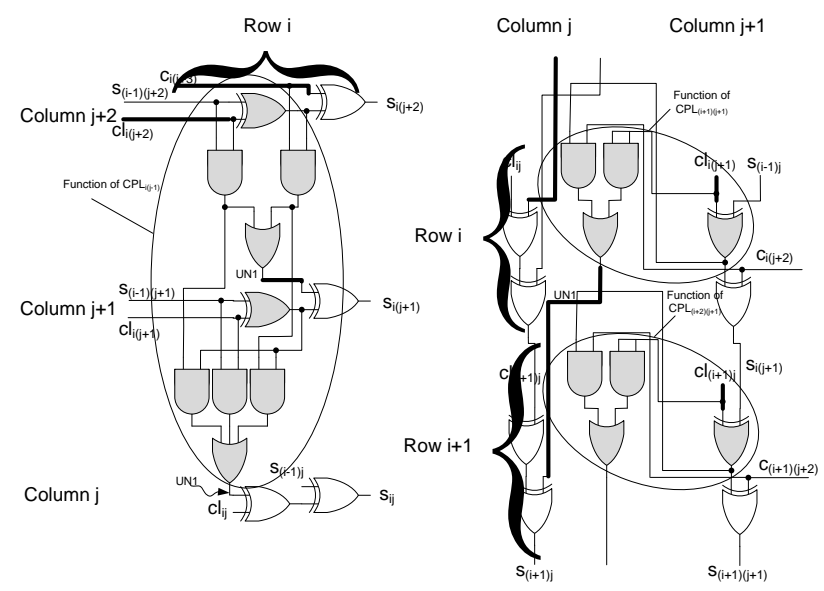

(a)

(b)

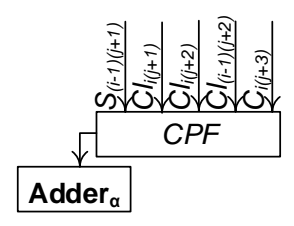

(c)

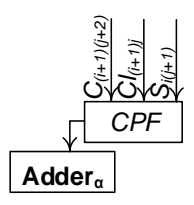

(d)
Figure 13. Two possible structures for CPLs: (a) using CLA adder, (b) using carry-save adder; corresponding CPF part in FBLA for Adder $_{\alpha}$ : (c) using CLA adder, (d) using carry-save adder.

In order to explain how Algorithm 6 extracts such optimized logic, let us consider an optimized version of the gate level implementation of restoring divider as shown in Fig. 14(a). As can be seen in the figure, $S_{G L} L_{1 j}$ and $\mathrm{CL}_{2 \mathrm{j}}$ in Fig. 8 are optimized together as a logic function. In order to extract such a combined $\mathrm{SGL}_{1 \mathrm{j}}$ and $\mathrm{CL}_{2 \mathrm{j}}$ logic, an XORbased mechanism discussed in the previous section is not efficient enough. To improve that mechanism, for each cell in the row $i$ and column $j$, CONE_verifier starts a forward searching from fan-outs. Suppose the algorithm starts from Column $j$ while higher columns are processed. By the first try, Gate 1 will be extracted as a cone with $c_{l(j+l)}$ and $d d_{l i}$ as its input. This cone cannot be used for updating FBLA. By the next try, Gates $1,5,3,6$, and 4 will be extracted. These gates with Gate 1 update FBLA by combining SGL and CL parts in Fig. 6. After that, this cone will be searched all over the design and every similar cone will be used to 
update FBLA. The final updated FBLA in this phase for the implementation in Fig. 14(a) is shown in Fig. 14(b) in which the combined SGL/CLs are shown by CNF. As it can be seen, in this step the sum generation and controlling modules (i.e., Adders and CFs in Fig. 6) are combined based on the data extracted from the implementation. This combination takes place whenever a detectable cone based on the combination of components in Fig. 6 is extracted.

After executing the CONE_verifier function, the unknown nodes remained from this step are processed as SCQC or SQC nodes in the next step. For example in Fig. 14(a) the highlighted node that is labeled as SCQC is the node that in this step is determined as output of CPL (i.e., Gate 7, 8, 9, 10, and 11). Based on the CPL extraction FBLA will be updated as is shown in Fig. 14(c). As it can be seen, updated CPF will be added to the FBLA that can be used for equivalence checking. It should be noted that the algorithm expects the optimization is uniform through the implementation. Then, when it determines a CPF in the beginning there is an expectation to have such functionality all through the network. Then, if structures with the expected inputs are extracted, the algorithm tries to apply equivalence checking on the updated FBLA.

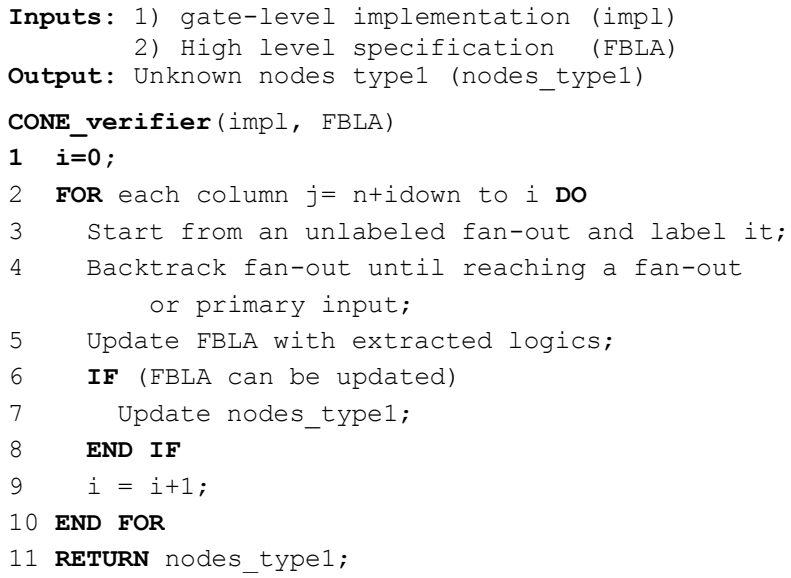

Algorithm 6. Cone verifier algorithm

\section{EXPERIMENTAL RESULTS}

In order to evaluate the proposed technique, we have applied it to several gate-level arithmetic circuits. Our algorithm has been implemented in $\mathrm{C}++$ and simulations are run on a personal computer having an Intel $2 \mathrm{GHz}$ Core 2 processor and $4 \mathrm{~GB}$ of main memory and running Windows 7. The gate-level circuits are synthesized by using commercial synthesis tools with various sorts of optimizations. We have compared the results for restoring, non-restoring, and SRT dividers with other related works, i.e., BDD-based techniques, combinational equivalence checking feature in $\mathrm{ABC}$ tool [6], and pure SAT solver [22]. In subsection VI1 , the results of verification and debugging when no optimizations are used in the implementation of the dividers are shown, while in subsection VI-2 the related results in the case of various optimizations are reported.

\section{Division Circuit Verification and Debugging}

In our first experiment, we realized the arithmetic function $F=C / D$ without deep optimization features where
SGLs could be extracted from the implementation. Therefore, the algorithm updated FBLA according to the FBLA shown in Fig. 6. The implementation also used ripple carry for CPL part. Several bugs have intentionally been injected into the gate level circuit. The bugs can be inverting an internal signal, changing the functionality of a two-input logic gate, or swapping an input signal of a two-input logic gate with another signal. Although such bugs are independently injected into the gate level, simultaneous bugs are also covered.

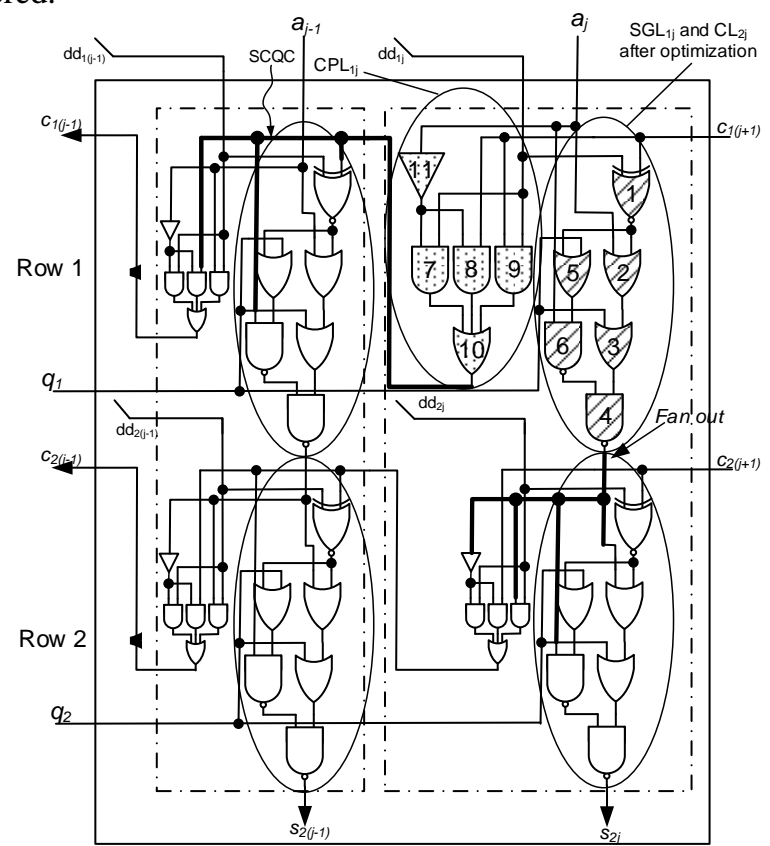

(a)

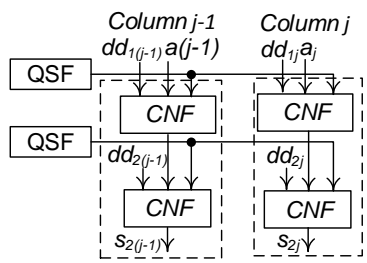

(b)

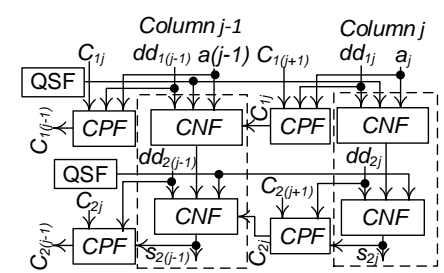

(c)
Figure 14. (a) An optimized gate-level implementation, (b) FBLA for the optimized gate-level implementation after CONE verifier function,

(c) FBLA for the optimized gate-level implementation after CPL extraction

The proposed debugging technique has been applied to the buggy implementation in order to detect and then correct errors. The results are summarized in major column "Without Optimization" of Tables 1, 2, and 3 where column $\# G$ gives the number of two-input gates and inverters. Column $\# B$ shows the number of simultaneous bugs injected into the circuits. Column $B S$ represents the location of injected bugs which consists of two cases: 1) SGL: bug injected into the sum generation logics and 2) CPLCLQSL: bug injected into the carry propagation, controlling or quotient selection logics.

Column $T_{0}$ indicates the time required to extract XOR terms (SGLs) for all columns. All run times are in seconds. Column $T_{l}$ refers to the time needed to extract and debugging other logics (CLs and CPLs). Note that the time needed to detect and replace bugs in the gate level circuit is included in $T_{1}$. As XOR extraction process initially takes place 
for all columns of FBLA, the buggy XORs will be detected really fast, and under such conditions, the algorithm stops proceeding through carry blocks. Regarding buggy XORs, the time required to detect bugs is negligible and the bug source is just reported to the user. On the other hand, when the bug source is not an XOR term, the debugging process will be dedicated to the realization of carry signals, and as a result, it becomes slower. The proposed algorithm can detect all injected bugs. The column $T_{2}$ shows the time for verifying bug-free circuits.

To compare our method with a BDD-based verification technique, the time needed for constructing BDD of each circuit is given in column $T_{3}$. BDDs are constructed using $\mathrm{ABC}[6]$ and time-out is set at 10800 seconds. Although $T_{3}$ has just reported the time required to construct BDDs of gate level circuits without checking the equivalence between two models, very large arithmetic circuits cannot be processed within 10800 seconds. By comparing $T_{2}$ and $T_{3}$, we observe that our method is much faster than BDD-based verification techniques, i.e., $267.7 \times, 246.1 \times$ and $293.1 \times$, average speedup for restoring, non-restoring and radix-2 SRT divisions, respectively. Note that average speedup is computed as $T_{3} /\left(T_{0}+T_{1}\right)$. It can be seen in the results that while the injected fault occurs in SGL the algorithm very quickly reports the buggy error and terminates. If, however, the bug is placed in CPL or CL, the algorithm takes more time for repairing the buggy circuit and continuing the debugging process.

We also compared the results with those of ABC [6]. For doing so, we used combinational equivalence checking (CEC) feature of $\mathrm{ABC}$ in order to compare two low level implementations. In CEC command, the behavior of primary inputs and primary outputs of two networks will be compared against each other. The CEC command of ABC is an improved version of SAT in which a hybrid approach based on fraiging and SAT solving is used to solve the mi- ter. Furthermore, the internal simulation needed for equivalence checking is improved to be more intelligent and, instead of circuit-based SAT solver, conjunctive normal form (CNF) is applied. This generally results in faster operation comparing to pure SAT solving technique. This way, first we generated a reference netlist from high-level specification and, after that, compared the netlist with the implementation. For example, for SRT radix-2 division algorithm, we generated a netlist with the simplest form of SRT division with ripple carry adders and totally separated components. After that we used this simple netlist as our reference model and compared the final implementation with this model. The time reported in column $T_{4}$ is the total time for this process. The average speedup obtained by the proposed method in comparison with $\mathrm{ABC}$ for restoring, non-restoring and radix-2 SRT dividers are 38.6x, 36.7x, and 50.1x, respectively.

In another experience, we used miniSAT as an SAT solver [22] to verify various divider circuits. In this experiment, like the previous one, first of all we generated a netlist reference model for division circuits and then we XORed the primary outputs of the two models. If the whole CNF is UNSAT that means two designs are equivalent. The results of the SAT solver have been shown in column $T_{5}$ of Tables 1,2 , and 3 . Obviously, the average speedup obtained by the proposed method in comparison with the SAT-based technique for restoring, non restoring and SRT division circuits are $38.1 \times, 33.9 \times$ and $53.3 \times$, respectively.

\section{Optimized Circuit Verification and Debugging}

In the second experiment, in order to illustrate the effectiveness of the modified debugging algorithm explained in Section V, we realize the arithmetic function $F=C / D$ when some optimizations have been taken into account. Again, several bugs have been intentionally injected into the gate level circuit and the results are summarized in major column "With Optimization" of Tables 1, 2, and 3.

TABLE 1 Debugging Results of “C / D" FOR RESTORING DivideR (CPU tIME IS GIVEN IN SECONDS)

\begin{tabular}{|c|c|c|c|c|c|c|c|c|c|c|c|c|c|c|c|c|c|c|}
\hline \multirow{2}{*}{$\begin{array}{l}\text { C / } \\
\text { D }\end{array}$} & \multicolumn{9}{|c|}{ Without Optimization } & \multicolumn{9}{|c|}{ With Optimization } \\
\hline & BS & $\begin{array}{l}\# \\
\text { B } \\
\end{array}$ & $\# \mathbf{G}$ & $\mathbf{T}_{0}$ & $\mathbf{T}_{1}$ & $\mathbf{T}_{2}$ & $\mathbf{T}_{3}$ & $\mathbf{T}_{4}$ & $\mathbf{T}_{5}$ & BS & $\begin{array}{l}\# \\
\text { B } \\
\end{array}$ & $\# \mathbf{G}$ & $\mathbf{T}_{0}$ & $\mathbf{T}_{1}$ & $\mathbf{T}_{2}$ & $\mathbf{T}_{3}$ & $\mathbf{T}_{4}$ & $\mathbf{T}_{5}$ \\
\hline \multirow{2}{*}{$9 / 5$} & CPLCLQSL & 2 & & 2.4 & 2.5 & 4.3 & \multirow{2}{*}{0.1} & \multirow{2}{*}{13.7} & \multirow[b]{2}{*}{12.1} & CPL & 2 & \multirow[b]{2}{*}{161} & 2.4 & 1.9 & 3.6 & \multirow[b]{2}{*}{0.09} & \multirow[b]{2}{*}{6.1} & \multirow[b]{2}{*}{3.2} \\
\hline & SGL & 2 & 196 & 0.7 & NA & NA & & & & SGLCL & 2 & & 2.1 & NA & NA & & & \\
\hline \multirow{2}{*}{$\begin{array}{c}13 / \\
7\end{array}$} & CPLCLQSL & 6 & \multirow{2}{*}{426} & 3.2 & 2.6 & 4.4 & \multirow[b]{2}{*}{0.4} & \multirow{2}{*}{18.3} & \multirow{2}{*}{16.6} & $\mathrm{CPL}$ & 6 & \multirow{2}{*}{355} & 4.6 & 2.1 & 5.3 & \multirow{2}{*}{0.32} & \multirow{2}{*}{12.3} & \multirow{2}{*}{12} \\
\hline & SGL & 1 & & 3 & NA & NA & & & & SGLCL & 1 & & 2.5 & NA & NA & & & \\
\hline \multirow{3}{*}{$\begin{array}{c}17 / \\
9\end{array}$} & CPLCLQSL & 7 & \multirow{3}{*}{744} & 6.1 & 4.9 & $\begin{array}{l}10 . \\
5\end{array}$ & \multirow{3}{*}{5.8} & \multirow{3}{*}{26.8} & \multirow{3}{*}{28} & CPL & 7 & 631 & 9.1 & 3.7 & 12. & \multirow{3}{*}{4.54} & \multirow{3}{*}{30.5} & \multirow{3}{*}{29.1} \\
\hline & & & & & & 5 & & & & & & (0) & & & 2 & & & \\
\hline & SGL & 3 & & 0.4 & NA & NA & & & & SGLCL & 3 & & 3.4 & NA & NA & & & \\
\hline \multirow{3}{*}{$\begin{array}{l}33 / 1 \\
7\end{array}$} & CPLCLQSL & 9 & \multirow{3}{*}{2596} & 11. & 9.8 & 22. & \multirow{3}{*}{ TO } & & & CPL & 9 & & 16. & 7.8 & 25. & & & \\
\hline & & & & 8 & & 3 & & 176 & 230. & & & 199 & 3 & & 5 & то & 201 & 212 \\
\hline & SGL & 3 & & 9.5 & NA & NA & & & & SGLCL & 3 & & 0.5 & NA & NA & & & \\
\hline & CPLCLOSL & 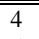 & & 20. & 22. & 39. & & & & CPL & 4 & & 29. & 15. & 45. & & & \\
\hline $65 / 3$ & & 5 & 1082 & 8 & 1 & 2 & TO & 2880 & 3003 & CPL & 5 & 892 & 7 & 1 & 2 & TO & 3550 & 3443 \\
\hline 3 & SGL & $\begin{array}{l}1 \\
2\end{array}$ & 3 & $\begin{array}{c}12 . \\
8\end{array}$ & NA & NA & 10 & & 5003 & SGLCL & $\begin{array}{l}1 \\
2\end{array}$ & 2 & $\begin{array}{c}19 . \\
8\end{array}$ & NA & NA & & & \\
\hline & Average speed & $\mathrm{p}(\mathrm{T}$ & versus ? & $\mathrm{T}_{4}$ a & & & $\begin{array}{c}267.7 \\
x\end{array}$ & $\begin{array}{c}38.6 \\
x\end{array}$ & $\begin{array}{c}38.1 \\
x\end{array}$ & Average & spee & up $\left(T_{2}\right.$ & rsus T & $\mathrm{T}_{4}$ and & & 235.3 & 4041.4 & 39.9 \\
\hline BS: B & urces; & & :nun & & $d b$ & & \#G: $\mathrm{n}$ & ber 0 & & $\mathrm{~N}$ & & cabl & & $\mathrm{T}_{0}=$ & & xtract c & $\frac{x}{\text { ined }}$ & \\
\hline $\begin{array}{l}\text { CL; T } \\
\text { BDD } \\
\text { second }\end{array}$ & $\begin{array}{l}=\text { Time to debug } \\
\text { ing } \mathrm{ABC} ; \mathrm{T}_{4}= \\
\end{array}$ & & $\begin{array}{l}\text { and QS } \\
\text { atained }\end{array}$ & $\mathrm{F}$ & fail t & nitiali & BLA; & & $\begin{array}{l}=\text { Tim } \\
\mathrm{T}_{5}=\mathrm{T}\end{array}$ & $\begin{array}{l}\text { verify bu } \\
\text { e obtained }\end{array}$ & fre & imples & ntation & & & $\begin{array}{r}\mathrm{T}_{3}=\mathrm{Ti} \\
\mathrm{TO}\end{array}$ & $\begin{array}{l}\text { to con } \\
\text { neout }\end{array}$ & $\begin{array}{l}\text { ruct } \\
10800\end{array}$ \\
\hline
\end{tabular}


TABLE 2 Debugging Resultsof "C / D" FOR Non Restoring Divider (CPu time IS GIVEN IN SECONDS)

\begin{tabular}{|c|c|c|c|c|c|c|c|c|c|c|c|c|c|c|c|c|c|c|}
\hline \multirow{2}{*}{ C / D } & \multicolumn{9}{|c|}{ Without Optimization } & \multicolumn{9}{|c|}{ With Optimization } \\
\hline & BS & \#B & \#G & $\mathbf{T}_{0}$ & $\mathbf{T}_{1}$ & $\mathbf{T}_{2}$ & $\mathbf{T}_{3}$ & $\mathbf{T}_{4}$ & $\mathbf{T}_{5}$ & BS & \#B & \#G & $\mathbf{T}_{\mathbf{0}}$ & $\mathbf{T}_{1}$ & $\mathbf{T}_{2}$ & $\mathbf{T}_{3}$ & $\mathbf{T}_{4}$ & $\mathbf{T}_{5}$ \\
\hline \multirow{2}{*}{$9 / 5$} & CPLCLQSL & 2 & \multirow{2}{*}{237} & 2.6 & & 4.8 & \multirow{2}{*}{0.07} & \multirow{2}{*}{10.5} & \multirow{2}{*}{9.1} & CPL & \multirow{2}{*}{4} & \multirow{2}{*}{231} & 2.6 & 2.9 & 4.8 & \multirow{2}{*}{0.05} & \multirow{2}{*}{19.1} & \multirow{2}{*}{21.8} \\
\hline & SGL & 2 & & 1.6 & NA & NA & & & & SGLCL & & & 1.6 & NA & NA & & & \\
\hline \multirow{2}{*}{$13 / 7$} & CPLCLQSL & 6 & \multirow{2}{*}{456} & 4.6 & 3.1 & 5.1 & \multirow{2}{*}{1.6} & \multirow{2}{*}{22.3} & \multirow{2}{*}{20.9} & CPL & \multirow{2}{*}{7} & \multirow{2}{*}{424} & 4.6 & 3.1 & 5.1 & \multirow{2}{*}{1.23} & \multirow{2}{*}{25.1} & \multirow{2}{*}{32.5} \\
\hline & SGL & 1 & & 3.1 & NA & NA & & & & SGLCL & & & 3.1 & NA & NA & & & \\
\hline \multirow{2}{*}{$17 / 9$} & CPLCLQSL & 7 & \multirow{2}{*}{753} & 8.2 & 7.4 & 13.4 & \multirow{2}{*}{135.4} & \multirow{2}{*}{31} & \multirow{2}{*}{29.7} & CPL & \multirow{2}{*}{10} & \multirow{2}{*}{711} & 8.2 & 7.4 & 13.4 & \multirow{2}{*}{135.4} & \multirow{2}{*}{33} & \multirow{2}{*}{35} \\
\hline & SGL & 3 & & 7.3 & NA & NA & & & & SGLCL & & & 7.3 & NA & NA & & & \\
\hline & CPLCLQSL & 9 & & 13.7 & 12.4 & 24.5 & & & & CPL & & & 13.7 & 12.4 & 24.5 & & & \\
\hline $33 / 17$ & SGL & 3 & 2649 & 4.3 & & & TO & 191.2 & 210 & SGLCL & 12 & 2023 & 4.3 & NA & NA & TO & 219 & 209 \\
\hline & CPLCLQSL & 45 & & 18.3 & 23.2 & 40.5 & & & & $\mathrm{CPL}$ & & & FA & FA & FA & & & \\
\hline $65 / 33$ & SGL & 12 & 11239 & 6.1 & NA & NA & TO & 2990 & 3003 & SGLCL & 57 & 9880 & FA & FA & FA & TO & 3033 & 3430 \\
\hline & Average spe & $\mathrm{p}(\mathrm{T}$ & versus 7 & $\mathrm{~T}_{4}$ an & & & $246.1 x$ & $36.7 x$ & $33.9 \mathrm{x}$ & Aver & e spe & $\operatorname{lup}\left(\mathrm{T}_{2}\right.$ & rsus $\mathrm{T}$ & $\mathrm{T}_{4}$ anc & & $2.4 \mathrm{x}$ & $5.3 x$ & $5.4 \mathrm{x}$ \\
\hline
\end{tabular}

TABLE 3 DEBUGging ResUltS OF "C / D" FOR SRT RADIX-2 DiVIDER (CPU TIME IS GIVEN IN SECONDS)

\begin{tabular}{|c|c|c|c|c|c|c|c|c|c|c|c|c|c|c|c|c|c|c|}
\hline \multirow{2}{*}{ C / D } & \multicolumn{9}{|c|}{ Without Optimization } & \multicolumn{9}{|c|}{ With Optimization } \\
\hline & BS & \#B & \#G & $\mathbf{T}_{\mathbf{0}}$ & $\mathbf{T}_{1}$ & $\mathbf{T}_{2}$ & $\mathbf{T}_{3}$ & $\mathbf{T}_{4}$ & $\mathbf{T}_{\mathbf{5}}$ & BS & \#B & \#G & $\mathbf{T}_{\mathbf{0}}$ & $\mathbf{T}_{1}$ & $\mathbf{T}_{2}$ & $\mathbf{T}_{3}$ & $\mathbf{T}_{4}$ & $\mathbf{T}_{5}$ \\
\hline \multirow{2}{*}{$9 / 5$} & CPLCLQSL & 2 & \multirow[b]{2}{*}{174} & 2 & 2.1 & 4.2 & \multirow[b]{2}{*}{0.1} & \multirow[b]{2}{*}{7.2} & \multirow[b]{2}{*}{6.5} & CPL & \multirow[b]{2}{*}{4} & \multirow{2}{*}{144} & 2.3 & 2 & 3.5 & \multirow{2}{*}{0.09} & \multirow[b]{2}{*}{6.5} & \multirow{2}{*}{7} \\
\hline & SGL & 2 & & 0.2 & NA & NA & & & & SGLCL & & & 2.1 & NA & NA & & & \\
\hline \multirow{2}{*}{$13 / 7$} & CPLCLQSL & 6 & \multirow{2}{*}{391} & 3 & 2.5 & 3.9 & \multirow{2}{*}{0.4} & \multirow{2}{*}{21.3} & \multirow{2}{*}{22.4} & CPL & \multirow{2}{*}{7} & \multirow{2}{*}{311} & 3.9 & 2 & 5.1 & \multirow{2}{*}{0.2} & \multirow{2}{*}{19.6} & \multirow{2}{*}{20.1} \\
\hline & SGL & 1 & & 0.2 & NA & NA & & & & SGLCL & & & 3 & NA & NA & & & \\
\hline \multirow{2}{*}{$17 / 9$} & CPLCLQSL & 7 & \multirow[b]{2}{*}{682} & 5.3 & 4.9 & 9.1 & \multirow{2}{*}{2.1} & \multirow{2}{*}{33.9} & \multirow{2}{*}{36.1} & CPL & \multirow[b]{2}{*}{10} & \multirow{2}{*}{608} & 8.3 & 3 & 10.9 & \multirow{2}{*}{2} & \multirow{2}{*}{39.5} & \multirow{2}{*}{40.2} \\
\hline & SGL & 3 & & 4.1 & NA & NA & & & & SGLCL & & & 3.3 & NA & NA & & & \\
\hline & CPLCLQSL & 9 & & 11.1 & 9.1 & 20.5 & & & & CPL & & & FA & FA & FA & & & \\
\hline $33 / 17$ & SGL & 3 & 2566 & 10.5 & NA & NA & TO & 246 & 248 & SGLCL & 12 & 1820 & FA & FA & FA & TO & 301 & 311 \\
\hline $65 / 33$ & CPLCLQSL & 45 & 9897 & 19.2 & 21.7 & 36 & TO & 3390 & 4001 & $\mathrm{CPL}$ & 57 & 8558 & 25.5 & 19.7 & 43.1 & TO & 1050 & 4301 \\
\hline 03155 & SGL & 12 & 9092 & 9.5 & NA & NA & 10 & 5390 & 4001 & SGLCL & 31 & OJJ0 & 19.9 & NA & NA & 10 & 4030 & 4501 \\
\hline & Average spee & up $(\mathrm{T}$ & ersus & $\mathrm{T}_{4}$ an & $\left.\mathrm{T}_{5}\right)$ & & $293.1 x$ & $50.1 x$ & $53.3 x$ & Avera & spe & up $\left(\mathrm{T}_{2}\right.$ & rsus & $\mathrm{T}_{4}$ an & & $256.8 \mathrm{x}$ & $52.5 x$ & $50.4 \mathrm{x}$ \\
\hline
\end{tabular}

As discussed in Section V, synthesis tools may combine some components together and generate optimized logic so that using FBLA shown in Fig. 6 is not possible. In such cases, according to Algorithm 6, we try to extract logics by back tracking from fan-out nodes and update FBLA by combining separated components in FBLA. In some cases, whenever the bug does not allow the algorithm to update FBLA, the algorithm will fail in its initialization phase that is shown by $F A$ in the tables. In such cases cone extraction cannot be mapped to a template in FBLA. This may be occurred by a bug or optimizations in which we are not able to find a single output cone for generating sum product as we have in our FBLA reference model. Practically, in most cases of optimization, SGLs can be extracted from the implementation. However, to change the implementation, we intentionally combined some parts in the HDL design to be synthesized more different. In the results SGL and CL were combined as Fig. 12(c) and FBLA of Fig. 14(b) was used. So some bugs were located in SGL/CL part and some other located in CPL part.

Column $T_{0}$ indicates the time required to extract combined components and initializing FBLA according to Algorithm 6. After making FBLA, the process of initializing is performed for all columns of FBLA. If some logics during this process are buggy, the algorithm stops proceeding through carry blocks and quickly reports the bug source for the user. If, however, any component is not buggy, the debugging process tries to check the carry propagation logics (CPLs) and also other logics if needed (CL/QSL). Column $T_{l}$ refers to the time needed to extract and then debug such logics. Column $T_{2}$ shows the time for verifying bug-free circuits. Again, by comparing $T_{2}$ and $T_{3}$, we observe that our method is much faster than BDD-based verification techniques, i.e., $235.3 \times, 2.4 \times$ and $256.8 \times$, average speedup for restoring, non-restoring and radix-2 SRT divisions, respectively. In addition, the average speedup compared to ABC are $41.4 \times, 5.3 \times$, and $52.5 \times$. And also the average speedup in comparison with SAT solver are 39.9×, 5.4×, and 50.4×.

The total number of injected bugs for optimized designs is the same as that for non optimized designs for better comparison. It can be seen for the same bugs, time required for verification and debugging of non optimized designs is shorter because in the case of optimized circuits it takes more time to update FBLA. The other reason is the longer back tracking time for FBLA initialization in optimized designs based on Algorithm 6. On the other hand, in the case of optimized circuits, after FBLA initialization, extracting 
CPLs, and QSLs take less time in comparison with extracting CPLs, CLs, and QSLs in non-optimized circuits.

\section{VII.CONCLUSION AND FUTURE WORKS}

In this paper, a debugging technique for optimized divider circuits has been proposed. The technique is based on a reverse-engineering mechanism of obtaining a high level model of the gate level implementation that is compared with a lower level of abstraction of a high level specification. If two models are not equivalent, the bugs will be automatically localized and then corrected with minimum user intervention. The proposed debugging algorithm is robust in terms of the run time so that two orders of magnitude speedup are obtained. A promising future work is to enhance this method to handle those optimization techniques which cause FBLA initialization fails. Moreover, the proposed technique can be enhanced to verify floatingpoint arithmetic circuits.

\section{ACKNOWLEDGEMENT}

This work was partially supported by Iran National Science Foundation (INSF) under Grant \#92018585.

\section{REFERENCES}

[1] R. E. Bryant, "Bit level analysis of an SRT divider circuit," in Proc. of Design Automation Conference, pp. 661-665, 1995.

[2] S. Y. Huang and K. T. Cheng, Formal Equivalence Checking and Design Debugging, Springer, June 1998.

[3] R. E. Bryant and Y.A. Chen, "Verifcation of arithmetic circuits with binary moment diagrams," in Proc. 32 $2^{\text {nd }}$ Design Automation Conference, pp. 535-541, June 1995.

[4] Y. Watanabe, N. Homma, T. Aoki, and T. Higuchi, "Arithmetic circuit verification based on symbolic computer algebra," in IEICE Transactions on Fundamentals of Electronics, Communications and Computer Sciences, vol. E91, pp. 3038-3046, Oct. 2008.

[5] O. Sarbishei, M. Tabandeh, B. Alizadeh and M. Fujita, "A formal approach for debugging arithmetic circuits," in IEEE Transactions on Computer-Aided Design of Integrated Circuits and Systems, vol. 28, no. 5, pp.742-754, May 2009.

[6] Berkeley Logic Synthesis and Verification Group, ABC: A System for Sequential Synthesis and Verification, Release 70930. http://www.eecs.berkeley.edu/ alanmi/abc.

[7] D. Cox, J. Little, and D. O'Shea, Ideals, varieties, and algorithms: An introduction to computational algebraic and geometry and commutative algebra, Springer, 2007.

[8] S. Vasudevan, V. Viswanath, R. Sumners, and J. A. Abraham, "Automatic verification of arithmetic circuits in RTL using stepwise refinement of term rewriting systems," in IEEE Transactions on Computers, vol. 56, no. 10, pp. 1401-1414, Oct. 2007.

[9] B. Parhami, Computer Arithmetic: Algorithms and Hardware Designs, $2^{\text {nd }}$ edition, Oxford University Press, New York, 2010.

[10] B. Alizadeh, and M. Fujita, "A Unified Framework for Equivalence Verification of Datapath Oriented Applications," in IEICE Transactions on Information and System, vol. E92-D, no. 5, pp. 985994, May 2009.

[11] D. Kroening, and S.A. Seshia, "Formal verification at higher levels of abstraction," in Proc. Intl. Conf. on Computer-Aided Design, pp. 572-578, 2007.

[12] E. Pavlenko, M. Wedler, D. Stoffel, and W. Kunz, "STABLE: A new QF-BV SMT solver for hard verification problems combining Boolean reasoning with computer algebra," in Proc. Design Automation and Test in Europe, pp. 1-6, March 2011.

[13] F. Farahmandi, and B. Alizadeh, "Groebner Basis based formal verification of large arithmetic circuits using Gaussian eliminiation and cone-based polynomial extraction," in Elsevier Microprocessors and MicroSystems - Embedded Hardware Design, vol 39, no. 2, pp. 83-96, March 2015.
[14] D. M. Russinoff, M. Kaufmann, E. Smith, and R. Sumners, "Formal Verification of Floating-Point RTL at AMD Using the ACL2 Theorem Prover," in Proc. IMACS World Congress on Scientific Computation, Applied Mathematics and Simulation, July 2005.

[15] H. Ruess, N. Shankar, and M. K. Srivas, "Modular verification of SRT division," in Journal of Formal Methods in System Design, vol. 14, issue 1, pp. 45-73, Jan 1999.

[16] B. Alizadeh, and M. Fujita, "Modular Datapath Optimization and Verification based on Modular-HED," in IEEE Transactions on Computer-Aided Design of Integrated Circuits and Systems, vol. 29, no. 9, pp. 1422-1435, Sep. 2010.

[17] Y. Chen, E. Clarke, P. Ho, Y. Hoskote, T. Kam, M. Khaira, J. O'Leary,and X. Zhao, "Verification of all circuits in a floating point unit using word-level model checking," in Proc. of Formal Methods in Computer-Aided Design, pp. 19-33, Nov. 1996.

[18] J. S. Moore, T. W. Lynch, and M. Kaufmann, "A mechanically checked proof of the AMD K-5 86 floating-point division program," in IEEE Trans. on Computer Engineering., 47(9):913-926, Sep. 1998.

[19] D. M. Russinoff, “A mechanically checked proof of IEEE compliance of the floating point multiplication, division and square root algorithms of the AMD-K7 processor," in Journal of Comp. Math., 1:148-200, 1998.

[20] R. Kaivola, R. Ghughal, N. Narasimhan, A. Telfer, J. Whittemore, S. Pandav, A. Slobodova, C. Taylor, V. Frolov, E. Reeber and A. Naik, "Replacing Testing with Formal Verification in Intel Corei7 Processor Execution Engine Validation," in Proc. Intl. Conf. on Computer Aided Verification, pp. 414-429, July 2009.

[21] A. Slobodova, J. Davis, S. Swords, and W. A. Hunt, "A flexible formal verification framework for industrial scale validation," in Proc. Intl. Conf. on Formal Methods and Models for Co-Design, pp. 89-97, June 2011.

[22] http://www.dwheeler.com/essays/minisat-user-guide.html

[23] M.H. Haghbayan, B. Alizadeh, P. Behnam and S. Safari, "Formal Verification and Debugging of Array Dividers with Auto-correction Mechanism," in Proc. Intl. Conf. on VLSI Design, pp. 80-85, 2014.

[24] A. Smith, A. Veneris, M. F. Ali, and A. Viglas, "Fault diagnosis and logic debugging using Boolean satisfiability," in IEEE Transactions on Computer-Aided Design of Integrated Circuits and Systems, vol.24, no.10, pp.1606-1621, Oct. 2005.

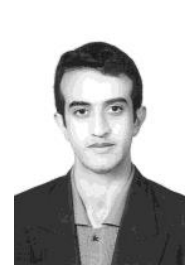

Mohammad Hashem Haghbayan received his BA from Ferdowsi University of Mashhad in 2005 and MS in 2008 from University of Tehran, Iran. His research interests include high-performance energy-efficient architectures, software-hardware microarchitecture interaction, power management techniques, and online/offline testing. $\mathrm{He}$ has several years of experience working in industry and designing IP cores as well as developing research tools.

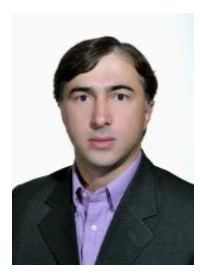

Bijan Alizadeh received the B.Sc., M.Sc. and Ph.D. degrees in Electrical and Computer Engineering from the University of Tehran, Iran in 1996, 1999 and 2004, respectively. Prior to joining the University of Tehran in 2010 as an assistant professor, he was an assistant professor at Sharif University of Technology from 2005 to 2007 and a Research Associate in VLSI Design and Education Center (VDEC), University of Tokyo from 2007 to 2010. He has developed many academic tools in verification, synthesis and other aspects of EDA. His current research interests include verification, test and synthesis of high level and system level designs, post silicon debugging, reconfigurable computing and embedded system design methodologies. 